\title{
Asset pricing in a Lucas fruit-tree economy with the best and worst in mind*
}

\author{
Alexander Zimper ${ }^{\dagger}$
}

November 23, 2011

\begin{abstract}
This paper studies a Lucas (1978) fruit-tree economy under the assumption that the agents are Choquet expected utility (CEU) rather than standard expected utility decision makers. More specifically, the agents' non-additive beliefs about the economy's dividend payment process are modelled as neo-additive capacities so that the agents' decision behavior emphasizes the best, respectively worst, possible economic scenarios. In contrast to existing models of Lucas-type economies with ambiguity averse agents (Epstein and Wang 1994), which ensure dynamic consistency through heavy restrictions on admissible ambiguity attitudes, my approach gives up dynamic consistency to the effect that quite general ambiguity attitudes become admissible. As the main formal result I establish the existence of a unique stationary equilibrium price function for this CEU Lucas economy. As the main economic insight I obtain that a representative agent who is rather preoccupied with the worst case scenario gives rise to a lower risk-free rate and a higher equity premium than predicted by the original expected utility Lucas economy. This difference is the greater the more surprising the economic information is that the agent receives.
\end{abstract}

Keywords: Choquet Expected Utility Theory, Portfolio Choice, Fat Tails, Asset Pricing Puzzles, Equity Premium, Risk-free Rate

JEL Classification Numbers: C62, D81, G11, G12

*I thank Jean-Philippe Lefort, Alexander Ludwig, Peter Wakker, two anonymous referees, and the coeditor Carl Chiarella for helpful comments and suggestions. Financial support from Economic Research Southern Africa (ERSA) is gratefully acknowledged.

${ }^{\dagger}$ Department of Economics, University of Pretoria, Private Bag X20, Hatfield 0028, South Africa. E-mail: alexander.zimper@up.ac.za 


\section{Introduction}

In a Lucas fruit-tree economy an infinitely lived representative agent trades in every time period assets that are claims to future dividend payments. Under the assumption that each time period's dividend payments are random variables with identical compact range that follow a time-homogenous Markov process, Lucas (1978) establishes existence and uniqueness of a stationary equilibrium price function. On the one hand, this seminal asset pricing model provides important insights into the relationship between the consumer's uncertainty with respect to future dividend payments and equilibrium asset prices. On the other hand, however, standard asset pricing models based on Lucas' approach predict equilibrium returns that are, by some large margin, at odds with actually observed asset returns. ${ }^{1}$ Lucas' approach rests on the decision-theoretic assumption that agents are subjective expected utility (EU) maximizers in the sense of Savage (1954). Since the EU assumption is systematically violated by real-life decision makers, it stands to hope that an asset pricing model that combines Lucas' fruit-tree economy with new developments in descriptive decision theory has more realistic appeal than the original approach.

This paper studies the Lucas fruit-tree economy under the decision-theoretic assumption that the agents may express ambiguity attitudes in violation of Savage's sure thing principle. To this end I describe the representative agent as a Choquet expected utility (CEU) rather than as an EU decision maker. As a generalization of EU theory, CEU theory is capable of accommodating ambiguity attitudes as elicited in paradoxes of the Ellsberg (1961) type by allowing for probability measures that are non-additive (Schmeidler 1986,1989; Gilboa 1987). CEU theory is formally equivalent to (cumulative) prospect theory (PT 1992) (Tversky and Kahneman 1992; Wakker and Tversky 1993) whenever PT 1992 is restricted to gains (for a comprehensive treatment of this equivalence see Wakker 2010). PT 1992, in turn, extends the celebrated concept of original prospect theory by Kahneman and Tversky (1979) to the case of several possible gain values in a way that satisfies first-order stochastic dominance.

More specifically, I focus attention on non-additive probability measures described as neo-additive capacities in the sense of Chateauneuf, Eichberger and Grant (2007) according to which an agent resolves his ambiguity by putting extra decision weights on the best, respectively worst, possible outcomes. As a consequence, the agents of this CEU Lucas economy have the best and the worst possible economic scenarios on

\footnotetext{
${ }^{1}$ For reviews on the rich literature on the equity premium and related asset return puzzles, which was initiated by the seminal contribution of Mehra and Prescott (1985), see the survey articles by Kocherlakota (1996), Campbell (2003), Mehra and Prescott (2003) and the textbook treatments in Cochrane (2001) and Duffie (2001).
} 
their minds when trading assets. Working with neo-additive capacities is attractive because they reduce the potential complexity of non-additive probability measures in a very tractable way such that important empirical features - e.g. inversely S-shaped probability transformation functions (see Chapter 11 in Wakker 2010) - are portrayed. ${ }^{2}$ The formal analysis in this paper demonstrates that CEU maximization with respect to neo-additive capacities remains technically tractable whereas this is hardly the case for general non-additive probability measures.

As this paper's main formal contribution I identify mathematical conditions that ensure the existence of a unique stationary equilibrium price function for the CEU Lucas economy. As a secondary formal contribution I characterize the resulting risk-free rate and the equity premium for the CEU Lucas economy. As the main economic insight I obtain that the assumption of neo-additive beliefs may contribute to a preference-based resolution of the risk-free rate and the equity premium puzzle (Mehra and Prescott 1985, 2003; Barberis and Thaler 2003). According to these puzzles Lucas-type models of assetpricing are at odds with empirically observed asset return distributions if, firstly, the risk-averse EU agents' concave vNM utility functions are realistically calibrated and, secondly, Muth's (1961) rational expectations assumption holds to the effect that the agents' subjective beliefs correctly reflect the empirical (i.e., objective) distributional parameters. In addition to expressing risk-attitudes modelled in terms of concave vNM utility functions, the agents of the CEU Lucas economy may express ambiguity by attaching extra decision weights to best, respectively worst, case scenarios. In particular, the assumption of overly pessimistic agents, i.e., agents that are preoccupied with the worst-case economic scenario, can apparently capture stylized facts discussed in the literature on asset-pricing puzzles that are ignored by the standard EU approach.

In contrast to EU models the standard long-run justification for Muth's rational expectations assumption does not apply to the CEU Lucas economy. Namely, as demonstrated in Zimper (2009, 2011) and in Zimper and Ludwig (2009), Bayesian learning under ambiguity does not - in general - converge to the underlying objective distribution when the agents observe large data-samples drawn from a stationary process. That is, the neo-additive beliefs of the agents in a CEU Lucas economy will stay bounded away from their objective counterparts to the effect that the agents may remain overly pessimistic in the long-run.

To generalize Lucas' original existence and uniqueness result to a CEU asset-pricing economy, I address the following conceptual and mathematical issues in this paper:

\footnotetext{
${ }^{2}$ To quote Peter Wakker (2010): "[...] the neo-additive functions are among the most promising candidates regarding the optimal tradeoff of parsimony and fit." (p. 209)
} 
1. For CEU decision makers a multitude of Bayesian update rules become possible so that an analytical solution will depend-unlike in the additive case on the chosen update rule. For illustrative reasons I focus on the full Bayesian update rule (Eichberger, Grant, and Kelsey 2006), which has nice empirical features.

2. As it turns out, the Markov property, according to which only the history in period $t$ is relevant for future beliefs in period $t+1$, is not easily satisfied but rather over-restrictive for conditional non-additive beliefs. Since the Markov property is essential for the existence of a stationary equilibrium, I abandon the perfect memory assumption to the effect that the stochastic process of dividend payments in my model does no longer constitute a filtration. Besides its technical convenience for a dynamic CEU model, the bounded memory assumption also has some intuitive appeal. Namely, instead of the infinitely lived representative agent of the original Lucas approach we can think of an infinite sequence of new generations of agents who all want to maximize their CEU from today's and tomorrow's consumption but who disregard the (statistical) information of their predecessors whenever it "lies way back in the past".

3. The agents of the CEU Lucas economy violate the principle of dynamic consistency to the effect that ex post agents may have a strict incentive to deviate from a portfolio choice that is optimal from an ex ante perspective. Consequently, a sensible equilibrium concept has to take account of the strategic situation in which different future agents play against each other. While Lucas' recursive equilibrium definition is equivalent to an Arrow-Debreu equilibrium if the commodity space includes all possible realizations of the dividend payment process, my-hybrid - definition of an equilibrium combines the market clearing condition of general equilibrium theory with the concept of subgame-perfect Bayesian Nash equilibria generalized to non-additive beliefs. Existing models of Lucas-type economies with ambiguity averse agents (i.e., rectangular multiple priors models based on Sarin and Wakker 1998b; Epstein and Schneider 2003) stick to the principle of dynamic consistency in order to admit for a technically tractable solution to the agent's portfolio maximization problem. More precisely, rectangular multiple priors models satisfy, by construction, a multiple priors version of the law of iterated expectations so that the standard $(=\mathrm{EU})$ technical tools for solving dynamic macroeconomic models go through. In Appendix I of this paper I formally demonstrate that such dynamically consistent models suffer from the conceptual drawback that they have to impose heavy (in my opinion: unrealistic) restrictions on admissible ambiguity attitudes. In contrast, the dynamically inconsistent CEU Lucas economy of 
this paper is capable of expressing quite general ambiguity attitudes whereby its analytical solution remains technically tractable due to the use of neo-additive capacities combined with the bounded memory assumption.

4. Recall that the expectation of a differentiable function $f$ with respect to an additive probability measure $\pi$ satisfies

$$
\frac{d E[f(x), \pi]}{d x}=E\left[\frac{d f(x)}{d x}, \pi\right] .
$$

Furthermore, the maximizer $x^{*}$ of this expectation is then uniquely characterized for strictly concave $f$ by the first-order condition

$$
\left.\frac{d E[f(x), \pi]}{d x}\right|_{x=x^{*}}=0 .
$$

Unfortunately, neither (1) nor (2) is guaranteed for the Choquet expectation with respect to non-additive probability measures, which is non-linear and which may not be differentiable everywhere. Under the assumptions of, firstly, neo-additive capacities and, secondly, a dividend space exhibiting a complete lattice structure with respect to the standard vector order, I am nevertheless able to characterize the agent's portfolio-optimization problem in terms of tractable first-order conditions. The reader should keep in mind, however, that any extension of my formal approach to more general non-additive probability measures or dividend spaces is not easily at hand and would amount to a highly non-trivial task.

The remainder of this analysis proceeds as follows. Section 2 discusses the relationship of my approach to the existing literature. In Section 3 I briefly review CEU theory and conditional neo-additive beliefs. Section 4 formally defines the information structure of the CEU Lucas economy. In Section 5 I prove existence and uniqueness of a stationary equilibrium price function. Section 6 characterizes the resulting risk-free rate and the equity premium in comparison to the standard EU economy. I conclude in Section 7 where I also provide an outlook on future research. Appendix I analyzes the formal relationship between Savage's sure-thing principle, on the one hand, and the principles of consequentialism and dynamic consistency, on the other hand. All mathematical proofs are relegated to Appendix II. 


\section{Relationship to the existing literature and further motivation}

This section starts out with a discussion about the relationship between this paper's model and the Simon Grant and John Quiggin argument in favor of governmental investments in private securities with the aim to drive the equity premium down. In a next step, I briefly review the rectangular max-min expected utility (MMEU) model of Sarin and Wakker (1998b) and Epstein and Schneider (2003) which is, to the best of my knowledge, the only decision theoretic model of ambiguity aversion that has been successfully applied to intertemporal models of asset pricing (the seminal article is Epstein and Wang 1994; for more recent models see Liu 2011; Miao and Wang 2011). Finally, I discuss the relationship of modelling ambiguity attitudes in terms of neo-additive capacities to the fat-tail approach.

Relationship to the Grant-Quiggin argument: Preferences versus market imperfections The fact that the assumption of CEU decision makers may contribute towards an explanation of the equity premium puzzle within a representative agent Lucas fruit tree economy bears implications on the discussion of whether government investments in risky projects are potentially welfare improving or not. In a series of highly original research articles (Grant and Quiggin 2002, 2003, 2005), Simon Grant and John Quiggin argue that government ownership of (selected) risky investment projects improves the ex ante welfare of a society if the equity premium is foremostly due to market imperfections rather than to individuals' preferences under uncertainty. While any detailed discussion of their approach is beyond the scope of this paper, the basic idea is straightforward. Namely, if the equity premium is rather preference-based, any government investment should remain - under suitable assumptions - neutral in a frictionless economy in the sense that the representative agent incorporates the government's investment as if it was his private investment to the effect that there cannot be any positive welfare effect (i.e., the equity premium remains as high as in the private investment case). In contrast, however, if the equity premium results from market imperfections, an efficient and benevolent government might be able to smooth out such imperfections through a combination of investments and taxation to the effect that welfare is strictly improved (i.e., the equity premium would be ideally driven down to zero through the elimination of risk). ${ }^{3}$

\footnotetext{
${ }^{3}$ For example, Grant and Quiggin (2003) consider an economy such that individuals cannot - due to adverse selection - fully insure themselves against the idiosyncratic risk of becoming unemployed in a recession. In this economy a government investment, mimicking private investments but being financed
} 
Of course, the question of whether real-life government investments in private projects are actually a good thing or not boils down to the question of whether government or market imperfections are the lesser evil. For instance, it appears unlikely that politicians - who care about ex post (re-) election - maximize ex ante welfare through the ex post introduction of a progressive tax scheme that may put off the (ex post) median voter. At this point, however, Grant and Quiggin argue that the sheer size of the equity premium suggests that it is caused by significant market imperfections rather than by preferences which, in turn, implies that government imperfections are the lesser evil. While such a conclusion may be compelling under the standard assumption of EU decision makers, my model suggests the possibility that the equity premium might be nevertheless based on preferences rather than on market imperfections whenever these preferences reflect ambiguity attitudes in the sense of CEU theory.

The focus of the present paper is on the technical conditions that ensure existence and uniqueness of an equilibrium price function under general model assumptions whereby I do not consider any specific calibration of the model parameters. That is, while my approach implies that the assumption of CEU decision makers may potentially contribute towards an explanation of asset pricing puzzles, it remains silent about the possible magnitude of such an explanation. In analogy to the original statement of the risk-free rate and equity premium puzzles - according to which Mehra and Prescott (1985) base realistic degrees of the concavity of vNM utilities on experimental findings that elicit values for the relative risk aversion parameter of a CRRA vNM utility function - it is a highly desirable avenue for future research to calibrate this paper's model with experimentally elicited values for the two additional preference parameters that come with neo-additive capacities, namely, an ambiguity- and a pessimism (optimism, respectively) under ambiguity parameter. Depending on the resulting values of the risk-free rate and the equity premium for such a calibrated CEU Lucas fruit-tree economy, the paper's model might either lend further support to Simon Grant's and John Quiggin's claim that the equity premium is mainly based on market-imperfections or it might make a case for a rather preference-based equity premium.

\section{Relationship to the rectangular MMEU approach}

Related to my approach are Epstein and Wang (1994) and, to a lesser extent ${ }^{4}$, Hansen,

through bonds, could strictly increase the ex ante expected utility of risk averse individuals if the bonds are paid back in the future through a progressive tax-scheme since such tax scheme would smooth out income differences across the complementary events of either being employed in the future or not.

${ }^{4}$ An agent in the robust control approach of Hansen, Sargent, and Tallarini (1999) fears possible misspecification errors in his model. Thus, while not directly developed within the MMEU framework of Gilboa and Schmeidler (1989), the decision situation of the period $t$ agents in the robust control 
Sargent, and Tallarini (1999) who also consider a Lucas fruit-tree economy under the assumption that the agents may express ambiguity attitudes by violating Savage's sure thing principle. Motivated by the MMEU ${ }^{5}$ approach of Gilboa and Schmeidler (1989), these authors consider agents who resolve their uncertainty not through a unique additive probability measure but rather through some set of additive probability measures. In contrast to my approach, however, these authors impose restrictions on possible ambiguity attitudes in order to satisfy dynamic consistency. For example, Epstein and Wang proceed with a recursive definition of the agents' utility functional such that they simply "[...] obviate the need for an updating rule" (p. 294, Epstein and Wang 1994) by taking conditional beliefs which satisfy the Markov property as primitives of their approach. In later contributions, Epstein and Schneider (2003), as well as Hansen, Sargent, Turmuhambetova, and Williams (2006) for a continuous time framework, consider proper MMEU preferences and provide a formal condition (rectangularity) on sets of priors such that the updated preferences obey dynamic consistency.

It is well known in the decision theoretic literature that the principles of consequentialism, dynamic consistency, and universalism ${ }^{6}$ give rise to Savage's subjective EU theory whenever dynamic decision situations are reduced - as in the MMEU approach - in a standard way to a static Savage framework for which ex ante and ex post preferences are defined over Savage acts (Sarin and Wakker 1998b; Ghirardato 2002). More precisely, Ghirardato (2002) proves that (universal) dynamic consistency combined with (universal) consequentialism is equivalent to the (universal) sure thing principle thereby implying EU theory under the remaining Savage axioms. As a consequence, any dynamic decision theoretic model that universally satisfies consequentialism as well as dynamic consistency within a static Savage framework is unable to express ambiguity attitudes.

To see the exact nature of ambiguity attitudes that can be expressed within a dynamically consistent MMEU model note that the rectangular MMEU concept goes back to Sarin and Wakker (1998b) who consider fixed decision trees. Sarin and Wakker (1998b, Theorem 2.1) already show that updating within the fixed information structure of a given tree satisfies consequentialism and dynamic consistency whenever the set of multiple priors is given as the reduced family of probability measures (=rectangular priors

approach can be re-interpreted as a max-min decision situation with respect to error-contaminated priors.

${ }^{5} \mathrm{MMEU}$ theory and CEU theory are related in the specific sense that a CEU representation with respect to a convex capacity is equivalent to a MMEU representation with respect to a set of priors given as the core of this capacity.

${ }^{6}$ In this context, the universalism principle states that behavioral axioms have to hold independently of any fixed information structure (i.e., for all possible events). For formal definitions of all three principles of dynamic decision making see Appendix I. 
in the terminology of Epstein and Schmeidler 2003). Similar to the fixed decision trees of Sarin and Wakker (1998b), Epstein and Schneider (2003) fix some information structure and assume that Savage's sure thing principle holds at selected events only. More precisely, in Appendix I of this paper I demonstrate that consequentialism and dynamic consistency hold for the rectangular MMEU model - in violation of the universalism principle exactly at all events that belong to the information structure generated by a fixed information partition of the ex post decision maker. On the one hand, the rectangular MMEU model does thus not reduce to standard EU theory because the sure thing principle does not hold as a universal principle. On the other hand, however, the sure thing principle holds in the rectangular MMEU model at all events that could be possibly observed by the decision maker. That is, the rectangular MMEU approach precludes, by construction, any possible dynamic version of the Ellsberg paradox through the exclusion of all events from the ex post decision maker's information structure for which the sure thing principle may be violated. In the words of Marciano Siniscalchi (2011):

"Epstein and Schneider's dynamic consistency requirement [...] implies that prior preferences must satisfy Savage's Postulate P2 relative to every conditioning event in the filtration under consideration." (p. 407)

As one implication of the rectangular MMEU model, individuals with different ex post information partitions must therefore have - in general - different sets of priors, i.e., ex ante ambiguity attitudes, to ensure dynamic consistency. In my opinion, universalism is very appealing because ex ante ambiguity attitudes should be conceptually independent of any specification of the ex post information structure. I therefore regard the concept of ambiguity attitudes admissible under the rectangular MMEU approach as too restrictive. For example, a decision maker who could observe any relevant event in the future has to reduce in the Epstein and Schneider model to the standard EU decision maker who does not exhibit any ex ante ambiguity attitudes.

Hansen et al. (2006) are also critical on the restriction of admissible sets of priors that results from imposing the rectangularity condition:

"If multiple priors truly are a statement of a decision maker's subjective beliefs, we think it is not appropriate to dismiss such beliefs on the grounds of dynamic inconsistency. Repairing that inconsistency through the enlargements necessary to induce rectangularity reduces the content of the original set of prior beliefs. In our context, this enlargement is immense, too immense to be interesting to us." (p. 78) 
Similarly, Eichberger, Grant, and Kelsey (2006) argue that ambiguity attitudes as elicited in Ellsberg paradoxes and inconsistencies in dynamic decision making are hardly separable and any attempt of doing so would be overtly restrictive. By giving up dynamic consistency, the agents of my CEU Lucas economy may express - in contrast to the rectangular MMEU model - ambiguity attitudes at all events, including events that may be observed ex post. It stands to hope that my approach may therefore capture relevant aspects of real-life decision making under ambiguity that are excluded by the Epstein and Schneider approach.

\section{Relationship to the fat tail approach}

There is a growing literature in financial economics (Tokat, Rachev, and Schwartz 2003; Bidarkota and McCulloch 2003; Bidarkota and Dupoyet 2007; Bidarkota, Dupoyet, and McCulloch 2009 and references therein) that studies asset-pricing models under the assumption that net, respectively gross, asset returns are described by fat tail rather than normal, respectively lognormal, distributions. The fat tail assumption thereby attempts to capture-within the standard framework of EU theory-the phenomenon that decision makers consider extreme events more likely than predicted by normal or lognormal distributions. As a drawback of this standard framework, however, Bidarkota and Dupoyet (2007) observe:

"[...] one reason why departures from a Gaussian distribution are not more common, despite its documented deficiencies, is the added complexity concomitant with such departures. Also, often in economic models, the use of many non-Gaussian distributions precludes the possibility of finding exact analytical solutions to equilibrium quantities of interest." (p.888)

By describing agents' subjective probability beliefs as neo-additive capacities this paper offers an alternative way — within the framework of CEU theory - for modelling the phenomenon that decision makers are preoccupied with the possibility of extreme events. Namely, the agents of my model attach additional subjective decision weights to extreme events whereby these non-additive decision weights arise from the agents' resolution of their ambiguity attitudes. As an analytical advantage of this approach, I derive a closedform solution that allows for comparative statics of asset-returns with respect to agents' attitudes towards optimism versus pessimism under ambiguity. Since this ambiguitybased approach towards fat tail phenomenons is founded on behavioral axioms that have proved useful in the description of real-life decision making, I feel that this is a decision-theoretically sound approach and not based on any behavioral ad-hoc assumptions. 


\section{Choquet decision theory}

This section briefly recalls basic elements of Choquet expected utility theory. CEU theory was first axiomatized by Schmeidler $(1986,1989)$ within the Anscombe and Aumann (1963) framework, which assumes preferences over objective probability distributions. Subsequently, Gilboa (1987) as well as Sarin and Wakker (1992) have presented CEU axiomatizations within the Savage (1954) framework, assuming a purely subjective notion of likelihood. When restricted to the domain of gains, CEU theory is formally equivalent to cumulative prospect theory (Tversky and Kahneman 1992; Wakker and Tversky 1993) which generalizes the celebrated prospect theory of Kahneman and Tversky (1979). Moreover, as a representation of preferences over lotteries, CEU theory coincides with rank dependent utility theory as introduced by Quiggin (1981, 1982). Within the context of CEU theory, properties of such capacities are used in the literature for formal definitions of ambiguity and uncertainty attitudes (Schmeidler 1989; Epstein 1999; Ghirardato and Marinacci 2002), pessimism and optimism (Eichberger and Kelsey 1999; Wakker 2001), as well as sensitivity to changes in likelihood (Wakker 2004, 2010).

\subsection{Neo-additive beliefs}

Consider a measurable space $(\Omega, \mathcal{F})$ with $\mathcal{F}$ denoting a $\sigma$-algebra on the state space $\Omega$ and a non-additive probability measure (=capacity) $\nu: \mathcal{F} \rightarrow[0,1]$ satisfying

(i) $\nu(\emptyset)=0, \nu(\Omega)=1$

(ii) $A \subset B \Rightarrow \nu(A) \leq \nu(B)$ for all $A, B \in \mathcal{F}$.

The Choquet integral of a bounded function $f: \Omega \rightarrow \mathbb{R}$ with respect to capacity $\nu$ is defined as the following Riemann integral extended to domain $\Omega$ (Schmeidler 1986):

$$
E[f, \nu(d \omega)]=\int_{-\infty}^{0}(\nu(\{\omega \in \Omega \mid f(\omega) \geq z\})-1) d z+\int_{0}^{+\infty} \nu(\{\omega \in \Omega \mid f(\omega) \geq z\}) d z
$$

whereby we will simply write $E[f, \nu]$ for $E[f, \nu(d \omega)]$. For example, assume that $f$ takes on $m$ different values such that $A_{1}, \ldots, A_{m}$ is the unique partition of $\Omega$ with $f\left(\omega_{1}\right)>$ $\ldots>f\left(\omega_{m}\right)$ for $\omega_{i} \in A_{i}$. Then the Choquet expectation (3) becomes

$$
E[f, \nu]=\sum_{i=1}^{m} f\left(\omega_{i}\right) \cdot\left[\nu\left(A_{1} \cup \ldots \cup A_{i}\right)-\nu\left(A_{1} \cup \ldots \cup A_{i-1}\right)\right] .
$$

My own approach focuses on non-additive probability measures that are defined as neo-additive capacities in the sense of Chateauneuf, Eichberger and Grant (2007). 
Definition. Fix some set of null-events $\mathcal{N} \subset \mathcal{F}$ for the measurable space $(\Omega, \mathcal{F}){ }^{7}$ The neo-additive capacity, $\nu$, is defined, for some $\delta, \lambda \in[0,1]$ by

$$
\nu(A)=\delta \cdot \nu_{\lambda}(A)+(1-\delta) \cdot \pi(A)
$$

for all $A \in \mathcal{F}$ such that $\pi$ is some additive probability measure satisfying

$$
\pi(A)= \begin{cases}0 & \text { if } A \in \mathcal{N} \\ 1 & \text { if } \Omega \backslash A \in \mathcal{N}\end{cases}
$$

and the non-additive probability measure $\nu_{\lambda}$ is defined as follows

$$
\nu_{\lambda}(A)= \begin{cases}0 & \text { iff } A \in \mathcal{N} \\ \lambda & \text { else } \\ 1 & \text { iff } \Omega \backslash A \in \mathcal{N} .\end{cases}
$$

Throughout this paper I restrict attention to sets of null-events $\mathcal{N}$ such that $A \in \mathcal{N}$ if and only if $\pi(A)=0$, which implies $\nu(A)=0$ (resp. $\nu(A)=1$ ) if and only if $\pi(A)=0$ (resp. $\pi(A)=1$ ). For any essential events, i.e., $A \notin \mathcal{N}$ and $\Omega \backslash A \notin \mathcal{N}$, the neo-additive capacity $\nu$ in (5) then simplifies to

$$
\nu(A)=\delta \cdot \lambda+(1-\delta) \cdot \pi(A)
$$

with $0<\pi(A)<1$. Neo-additive capacities can thus be interpreted as non-additive beliefs that stand in for deviations from additive beliefs such that a parameter $\delta$ (degree of ambiguity) measures the lack of confidence the decision maker has in some subjective additive probability distribution $\pi$. The following proposition extends a result (Lemma 3.1) of Chateauneuf, Eichberger and Grant (2007) for finite random variables to the more general case of random variables with a bounded range.

Observation 1. Let $f: \Omega \rightarrow \mathbb{R}$ be a $\mathcal{F}$-measurable function with compact range. The Choquet expected value (3) of $f$ with respect to a neo-additive capacity (5) is then given by

$$
E[f, \nu]=\delta(\lambda \max f+(1-\lambda) \min f)+(1-\delta) E[f, \pi] .
$$

\footnotetext{
${ }^{7}$ Intuitively speaking, null events are the events that the decision maker deems impossible. For instance, within a Savage framework we have that $A \in \mathcal{N}$ if and only if, for all Savage acts $f, g, h, h^{\prime}$,

$$
h_{A} f \succeq h_{A}^{\prime} g \Leftrightarrow f \succeq g,
$$

i.e., consequences on $A$ are irrelevant to the decision maker; (see Appendix I for the notation).
} 
According to Observation 1, the Choquet expected value of a random variable $f$ with respect to a neo-additive capacity is a convex combination of the expected value of $f$ with respect to some additive probability measure $\pi$ and an ambiguity part. If there is no ambiguity, i.e., $\delta=0$, then the Choquet expected value (9) reduces to the standard expected value of a random variable with respect to an additive probability measure. In case there is some ambiguity, however, the second parameter $\lambda$ measures how much weight the decision maker puts on the best possible outcome of $f$ when resolving his ambiguity. Conversely, $(1-\lambda)$ is the weight he puts on the worst possible outcome of $f$. As a consequence, one can interpret $\lambda$ as an "optimism under ambiguity" parameter whereby $\lambda=1$, resp. $\lambda=0$, corresponds to extreme optimism, resp. extreme pessimism, with respect to resolving ambiguity in the decision maker's belief.

Remark. In addition to the above - motivational-interpretation, Peter Wakker (2010, Equ 7.2.6) offers an alternative - cognitive - interpretation for the parameters of neo-additive capacities according to which $\delta$ is an index of likelihood insensitivity whereas $(2 \delta \lambda+(1-\delta)) / 2$ is an index of elevation. Wakker (2010) refers to likelihood insensitivity as a new psychological concept which "reflects diminishing sensitivity for a scale bounded from two sides" (p. 227). According to this cognitive interpretation, agents who express ambiguity in my model might be alternatively interpreted as agents who do not sufficiently distinguish between probabilities that lie between zero and one (e.g., individuals who would resolve any uncertainty in a fifty-fifty way).

\subsection{Bayesian updating of neo-additive beliefs}

CEU theory has been developed in order to accommodate paradoxes of the Ellsberg type which show that real-life decision-makers violate Savage's sure thing principle. For the interested reader I demonstrate in Appendix I in some detail that the abandoning of the sure thing principle has two important implications for conditional CEU preferences over Savage-acts. Firstly, in contrast to Bayesian updating of additive probability measures, there exist several perceivable Bayesian update rules for non-additive probability measures (Gilboa and Schmeidler 1993; Sarin and Wakker 1998a; Pires 2002; Eichberger, Grant and Kelsey 2006; Siniscalchi 2011). Secondly, if CEU preferences are updated in accordance with an updating rule that (universally) satisfies the principle of consequen-

tialism, then these CEU preferences violate the principle of dynamic consistency (in a universal sense) whenever they do not reduce to EU preferences.

In the present paper I assume that the agents form conditional capacities in accor- 
dance with the full Bayesian update rule such that, for all non-null $A, B \in \mathcal{F}$,

$$
\nu(A \mid B)=\frac{\nu(A \cap B)}{\nu(A \cap B)+1-\nu(A \cup \neg B)} .
$$

Since the full Bayesian update rule satisfies consequentialism (Appendix I), the CEU preferences of my model violate dynamic consistency. An application of (10) to a neoadditive capacity results, for non-null $A, B$, in

$$
\begin{aligned}
\nu(A \mid B) & =\frac{\delta \cdot \lambda+(1-\delta) \cdot \pi(A \cap B)}{\delta \cdot \lambda+(1-\delta) \cdot \pi(A \cap B)+1-(\delta \cdot \lambda+(1-\delta) \cdot \pi(A \cup \neg B))} \\
& =\frac{\delta \cdot \lambda}{\delta+(1-\delta) \cdot \pi(B)}+\frac{(1-\delta)}{\delta+(1-\delta) \cdot \pi(B)} \pi(A \cap B),
\end{aligned}
$$

which gives rise to the following observation.

Observation 2. Suppose that the non-additive belief of the agent is given as the neoadditive capacity (5). If the agent applies the full Bayesian update rule, his conditional neo-additive belief is given as

$$
\nu(A \mid B)=\delta_{B} \cdot \lambda+\left(1-\delta_{B}\right) \cdot \pi(A \mid B),
$$

for non-null $A, B \in \mathcal{F}$ whereby

$$
\delta_{B}=\frac{\delta}{\delta+(1-\delta) \cdot \pi(B)}
$$

For the interested reader I review in Appendix I two alternative update rules for non-additive probability measures; namely, the optimistic and pessimistic update rules (Gilboa and Schmeidler 1993). Compared to these update rules, the full Bayesian update rule is less extreme and has therefore more empirical appeal. For example, Cohen, Gilboa, Jaffray, and Schmeidler (2000) investigate the question whether the pessimisticin their terms: the maximum likelihood (=ML) — update rule or the full Bayesian $(=\mathrm{FB})$ update rule is consistent with ambiguity averse subjects' choice behavior. Their experimental findings establish an approximate ratio of 2:1 in favor for the full Bayesian update rule. More specifically, Cohen et al. (2000) write:

"Thus, out of the remaining 33 subjects who must have multiple priors in sample I, 12 chose in accordance with MLU prediction (36, 36\%), whereas 19 of them made choices consistent with FBU (57, 58\%), and 2 were inconsistent with either theory $(6,06 \%)$. Results in sample II are similar: out of 43 subjects, MLU accounts for the choices of 10 of them (23, 25\%), FBU for the choices of 23 others ( $53,5 \%$ ), whereas the choices of the remaining 10 (23, 25\%) are unexplained." (p. 130) 


\section{Information structure and assumptions on beliefs}

A Lucas fruit-tree economy is an infinite horizon representative agent economy with $k$ different assets. In a CEU Lucas fruit-tree economy every period $t$ agent is a Choquet decision maker who is, firstly, uncertain about future dividend-payments of these assets but has, secondly, made some observation about previous dividend payments. In contrast to the standard approach according to which a period $t$ agent has observed all period $0, \ldots, t$ dividend payments, I restrict attention to a situation of bounded memory where every period $t$ agent has only observed the present period's dividend payment. On the one hand, this restriction is for technical convenience since, under the assumption of a perfect memory, full Bayesian updating of neo-additive capacities is in conflict with the stationarity ensuring Markov property, (for technical details see the remark at the end of this section). On the other hand, this memory restriction is also closer to the arguably more realistic situation in which not an infinitely lived representative agent will be trading assets forever but rather in which new generations of agents are constantly pouring in whereby their attention spans for past observations are limited. As a consequence, the agents of my model only care about a fixed number of past periods. ${ }^{8}$ Loosely speaking, the bounded memory assumption brings thus some flavour from overlapping generations models - the other working horse of dynamic macroeconomics - into the Lucas fruit-tree economy.

In what follows we construct a non-additive probability space $(\nu(\cdot \mid \cdot), \Omega, \mathcal{F})$ that captures the above assumptions on the information structure. Formally, let $\mathbf{Y}_{t}$ with generic element $\mathbf{y}_{t}=\left(y_{1, t}, \ldots, y_{k, t}\right)$ denote the set of possible dividend payments in period $t$, whereby I assume that $\mathbf{Y}_{t}=\mathbf{Y}$ for $t=0,1, \ldots$ such that $\mathbf{Y}$ is a non-empty compact subset of the Euclidean space $\mathbb{R}^{k}$. As the relevant state space I define

$$
\Omega=\times_{s=0}^{\infty} \mathbf{Y}
$$

with generic element $\omega=\left(\mathbf{y}_{1}, \mathbf{y}_{2}, \ldots\right)$. By this definition of the state space, any relevant uncertainty in the model will boil down - in terms of adopted stochastic processes such as equilibrium prices - to the agents' uncertainty about the assets' dividend payment streams. In line with the bounded memory assumption let us define the information partition of the period $t$ agent is given as the following collection of subsets of $\Omega$

$$
\mathcal{P}_{t}=\left\{\left(\times_{s=0}^{t-1} \mathbf{Y}\right) \times\left\{\mathbf{y}_{t}\right\} \times\left(\times_{s=t+1}^{\infty} \mathbf{Y}\right)\right\}_{\mathbf{y}_{t} \in \mathbf{Y}}
$$

\footnotetext{
${ }^{8}$ While I restrict - out of notational transparency - attention to the special case of only one previous observation (i.e., $n=0$ ), the formal analysis would be equivalent for the more general case where every period $t$ agent has made $n+1$ previous observations, i.e., $t-n, \ldots, t$. That is, stationarity is ensured in my framework whenever the number of past observations, $n$, is constant for all $t$ agents.
} 
That is, the period $t$ agent can only observe the most recent dividend payments, given by $\mathbf{y}_{t}$, whereas he ignores/forgets dividend payments that lie further in the past, (formally, by just "observing" the event $\times_{s=0}^{t-1} \mathbf{Y}$ any past dividend payment stream $\mathbf{y}_{0}, \ldots, \mathbf{y}_{t-1} \in$ $\times_{s=0}^{t-1} \mathbf{Y}$ is regarded as possible by the period $t$ agent). Here, we restrict attention to the special case of one previous observation for the sake of notational transparency; namely, the formal analysis would be completely equivalent for the more general case

$$
\mathcal{P}_{t}^{\prime}=\left\{\left(\times_{s=0}^{t-n-1} \mathbf{Y}\right) \times\left\{\mathbf{y}_{t-n}\right\} \times \ldots \times\left\{\mathbf{y}_{t}\right\} \times\left(\times_{s=t+1}^{\infty} \mathbf{Y}\right)\right\}_{\mathbf{y}_{t} \in \mathbf{Y}}
$$

where every period $t$ agent has made $n+1$ previous observations with $n \geq 0$. That is, stationarity is ensured in my framework whenever the number of past observations, $n$, is constant for all period $t$ agents.

Denote by $\mathcal{F}_{t}$ the Borel $\sigma$-algebra generated by $\mathcal{P}_{t}$, that is, $\mathcal{F}_{t}$ is the smallest $\sigma$-algebra containing all sets

$$
\left(\times_{s=0}^{t-1} \mathbf{Y}\right) \times A \times\left(\times_{s=t+1}^{\infty} \mathbf{Y}\right)
$$

in $\Omega$ such that $A$ is an open set in $\mathbf{Y}$. Intuitively speaking, $\mathcal{F}_{t}$ contains all events that can be observed/learnt by the period $t$ agent. For example, if the agent learns that $\mathbf{y}_{t}=\$ 10$, then he also learns, among other things, that the period $t$ dividend payment lies in the open interval $A=(\$ 5, \$ 20)$.

Denote by $\mathcal{F}_{\infty}$ the standard product algebra generated by $\mathcal{F}_{1}, \mathcal{F}_{2}, \ldots$. Define now the $\mathcal{F}_{t}$-measurable coordinate random variables $Y_{j, t}, j=1, \ldots, k$, such that

$$
Y_{j, t}(\omega)=y_{j, t}
$$

and the corresponding $\mathcal{F}_{t}$-measurable coordinate random vector $\mathbf{Y}_{t}$ such that

$$
\mathbf{Y}_{t}(\omega)=\mathbf{y}_{t}
$$

whereby $\mathbf{y}_{t}=\left(y_{1, t}, \ldots, y_{k, t}\right)$ is interpreted as the realized period $t$ dividend payments in the state of the world $\omega$. That is, $\mathbf{Y}_{t}$ stands for the random period $t$ dividend payments, which are observable by the period $t$ agent. Observe that, by construction of the state space, $\max \mathbf{Y}_{t}$ and $\min \mathbf{Y}_{t}$ are well defined with respect to the standard vector order whereby I introduce - for later reference - the following notational convention

$$
\overline{\mathbf{y}} \equiv\left(\bar{y}_{1}, \ldots, \bar{y}_{k}\right)=\max \mathbf{Y}_{t} \text { and } \underline{\mathbf{y}} \equiv\left(\underline{y}_{1}, \ldots, \underline{y}_{k}\right)=\min \mathbf{Y}_{t}
$$

That is, $\overline{\mathbf{y}}$ denotes the best and $\underline{\mathbf{y}}$ denotes the worst possible economic scenario in any given time period according to which every asset pays out its maximal, resp. minimal, dividend.

Let us fix the full Bayesian update rule (10) so that the conditional neo-additive capacity in $(\nu(\cdot \mid \cdot), \Omega, \mathcal{F})$ is given by $(13)$. A period $t$ agent's belief about future 
dividend payments conditional on the period $t$ payment $\mathbf{y} \in \mathbf{Y}$ is then defined as the finite-dimensional conditional neo-additive capacity $\nu_{t+1, \ldots, t+1+n}(\cdot \mid \cdot)$ such that, for any Borel sets $A_{t+1}, \ldots, A_{t+1+n}$,

$$
\begin{aligned}
& \nu_{t+1, \ldots, t+1+n}\left(A_{t+1} \times \ldots \times A_{t+1+n} \mid \mathbf{y}\right) \\
= & \nu\left(\left(\times_{s=0}^{t-1} \mathbf{Y}\right) \times A_{t+1} \times \ldots \times A_{t+1+n} \times\left(\times_{s=t+2+n}^{\infty} \mathbf{Y}\right) \mid\left(\times_{s=0}^{t-1} \mathbf{Y}\right) \times\{\mathbf{y}\} \times\left(\times_{s=t+1}^{\infty} \mathbf{Y}\right)\right)
\end{aligned}
$$

Formally, the period $t$ agent thus bases his conditional beliefs about future dividend payments exclusively on information about the period $t$ dividend payment.

Finally, let us impose two assumptions on $(\nu(\cdot \mid \cdot), \Omega, \mathcal{F})$. Firstly, we require that all finite-dimensional conditional capacities $\nu_{t+1}(\cdot \mid \cdot)$ are independent of $t$. Secondly, we need a technical condition, the "Feller property", which ensures that the conditional expectations operator will map the space of bounded real-valued continuous function on Y into itself. Intuitively speaking, the "Feller property" works as a continuity condition which ensures that small changes (in the Euclidean sense) in the observed dividend payments only result in small changes (also in the Euclidean sense) in the conditional expectation of random variables that continuously depend on these dividend payments.

\section{Assumptions on beliefs}

(A1) "Time-homogeneity": For all $t \geq 0$ and all $\mathbf{y} \in \mathbf{Y}$,

$$
\nu_{t+1}(A \mid \mathbf{y})=\delta_{\mathbf{y}} \lambda+\left(1-\delta_{\mathbf{y}}\right) \pi(A \mid \mathbf{y})
$$

for all Borel sets $A$ in $\mathbf{Y}$ whereby

$$
\delta_{\mathbf{y}}=\frac{\delta}{\delta+(1-\delta) \cdot \pi(\mathbf{y})}
$$

(A2) "Feller property": Let $f: \mathbf{Y} \rightarrow \mathbb{R}$ be any real-valued continuous function in $\mathbf{Y}$. Define $T f: \mathbf{Y} \rightarrow \mathbb{R}$ such that

$$
(T f)(\mathbf{y})=\delta_{\mathbf{y}}\left(\lambda \max _{\mathbf{y}^{\prime} \in \mathbf{Y}} f\left(\mathbf{y}^{\prime}\right)+(1-\lambda) \min _{\mathbf{y}^{\prime} \in \mathbf{Y}} f\left(\mathbf{y}^{\prime}\right)\right)+\left(1-\delta_{\mathbf{y}}\right) E\left[f, \pi\left(d \mathbf{y}^{\prime} \mid \mathbf{y}\right)\right] .
$$

Then $T f$ is also a real-valued continuous function in $\mathbf{Y}$.

According to the standard approach, agents have perfect memory so that the information partition of the period $t$ agent is given as

$$
\mathcal{P}_{t}=\left\{\left\{\left(\mathbf{y}_{0}, \ldots, \mathbf{y}_{t}\right)\right\} \times\left(\times_{s=t+1}^{\infty} \mathbf{Y}\right)\right\}_{\left(\mathbf{y}_{0}, \ldots, \mathbf{y}_{t}\right) \in \times_{s=0}^{t} \mathbf{Y}}
$$


That is, the agents' information partitions are getting finer with increasing $t$, implying that the agent never forgets any previous observations. Accordingly, the $\mathcal{F}_{t}$ generated by the $\mathcal{P}_{t}$ constitute a filtration, i.e., $\mathcal{F}_{1} \subseteq \mathcal{F}_{2} \subseteq \ldots \subseteq \mathcal{F}$. To ensure stationary, standard EU models stipulate that the period $t$ agents' beliefs satisfy the one-step-ahead Markov property according to which for any observed history $\left(\mathbf{y}_{0}, \ldots, \mathbf{y}_{t}\right) \in \times_{i=0}^{t} \mathbf{Y}$,

$$
\pi_{t+1}\left(A_{t+1} \mid \mathbf{y}_{0}, \ldots, \mathbf{y}_{t}\right)=\pi_{t+1}\left(A_{t+1} \mid \mathbf{y}_{t}\right)
$$

where

$$
\begin{aligned}
& \pi_{t+1}\left(A_{t+1} \mid \mathbf{y}_{0}, \ldots, \mathbf{y}_{t}\right) \\
= & \pi\left(\left(\times_{s=0}^{t-1} \mathbf{Y}\right) \times A_{t+1} \times\left(\times_{s=t+2+n}^{\infty} \mathbf{Y}\right) \mid\left\{\left(\mathbf{y}_{0}, \ldots, \mathbf{y}_{t}\right)\right\} \times\left(\times_{s=t+1}^{\infty} \mathbf{Y}\right)\right)
\end{aligned}
$$

for the additive probability space $(\pi, \Omega, \mathcal{F})$. For additive beliefs the Markov property has therefore formally the same effect as a restricted memory process: the same stationary environment arises in case the agent ignores previous observations because they are not relevant (=Markov property) or in case the agent simply lacks these observations (=restricted memory).

Unfortunately, this is no longer the case for non-additive probability measures. If we had adopted the perfect-memory assumption, the Markov-property would require for all Borel-sets $A$ and all $\mathbf{y}_{0}, \ldots \mathbf{y}_{t-1}$ and all $\mathbf{y}$

$$
\begin{aligned}
\nu\left(A \mid \mathbf{y}_{0}, \ldots \mathbf{y}_{t-1}, \mathbf{y}\right) & =\nu(A \mid \mathbf{y}) \\
& \Leftrightarrow \\
\delta_{\mathbf{y}_{0}, \ldots \mathbf{y}_{t-1}, \mathbf{y}} \cdot \lambda+\left(1-\delta_{\mathbf{y}_{0}, \ldots \mathbf{y}_{t-1}, \mathbf{y}}\right) \cdot \pi\left(A \mid \mathbf{y}_{0}, \ldots \mathbf{y}_{t-1}, \mathbf{y}\right) & =\delta_{\mathbf{y}} \cdot \lambda+\left(1-\delta_{\mathbf{y}}\right) \cdot \pi(A \mid \mathbf{y})
\end{aligned}
$$

with

$$
\begin{aligned}
\delta_{\mathbf{y}_{0}, \ldots \mathbf{y}_{t-1}, \mathbf{y}} & =\frac{\delta}{\delta+(1-\delta) \cdot \pi\left(\mathbf{y}_{0}, \ldots \mathbf{y}_{t-1}, \mathbf{y}\right)} \\
& >\frac{\delta}{\delta+(1-\delta) \cdot \pi(\mathbf{y})} \\
& =\delta_{\mathbf{y}}
\end{aligned}
$$

since (for non-degenerate cases)

$$
\pi\left(\mathbf{y}_{0}, \ldots \mathbf{y}_{t-1}, \mathbf{y}\right)<\pi(\mathbf{y}) .
$$

As a consequence, the system of equalities (29) imposes very heavy restrictions on the additive probability measure $\pi$ and parameter-values $\delta$ and $\lambda$. For example, any additive probability measure $\pi$ satisfying

$$
\pi\left(A \mid \mathbf{y}_{0}, \ldots \mathbf{y}_{t-1}, \mathbf{y}\right)=\pi(A \mid \mathbf{y})
$$

or even independence would be excluded by (29). By the bounded memory assumption, these rather implausible restrictions on admissible beliefs are avoided. 


\section{Existence and uniqueness of the equilibrium}

This section states and proves this paper's main formal result which establishes the existence of a unique stationary asset-pricing equilibrium for the CEU Lucas economy. The formal proof is based on a contraction mapping argument that is similar to Lucas' formal argument in the case of EU agents with additive beliefs.

Consider the decision-situation of a representative period $t$ agent who has initial endowment $\mathbf{z}_{t}=\left(z_{1, t}, \ldots, z_{k, t}\right) \in[0,1]^{k}$ of $k$ different assets whereby $\mathbf{z}_{0}=\mathbf{1}$. The agent has to choose the amounts of assets he is going to buy, respectively sell, on the period $t$ spot market; that is, he effectively decides about his period $t+1$ asset holdings $\mathbf{z}_{t+1} \in$ $[0,1]^{k}$. For realized dividend payments $\mathbf{y}_{s}=\left(y_{1, s}, \ldots, y_{k, s}\right) \in \mathbb{R}^{k}$ per asset unit and exdividend spot market asset prices $\mathbf{p}_{s}=\left(p_{1, s}, \ldots, p_{k, s}\right) \in \mathbb{R}^{k}$, the period $s=t, t+1, \ldots$ consumption from the agent's perspective is given as follows

$$
c\left(\mathbf{y}_{s}, \mathbf{p}_{s}, \mathbf{z}_{s}, \mathbf{z}_{s+1}\right)=\mathbf{y}_{s} \cdot \mathbf{z}_{s}+\mathbf{p}_{s} \cdot\left(\mathbf{z}_{s}-\mathbf{z}_{s+1}\right) .
$$

Let us stick to the standard assumption of additively time-separable preferences so that, for a given state of the world $\left(\mathbf{y}_{1}, \ldots, \mathbf{y}_{t}, \mathbf{y}_{t+1}, \ldots\right) \in \Omega$, the period $t$ agent gains vNM utility

$$
U\left(\mathbf{z}_{t+1}\right)=\sum_{s=t}^{\infty} \beta^{s-t} u\left(c\left(\mathbf{y}_{s}, \mathbf{p}_{s}, \mathbf{z}_{s}, \mathbf{z}_{s+1}\right)\right)
$$

from choosing $\mathbf{z}_{t+1}$ while $\mathbf{z}_{t}, \mathbf{z}_{s}$, with $s>t+1$, and $\mathbf{p}_{s}$, with $s \geq t$, are fixed. Here, $\beta \in(0,1)$ denotes the time-discount factor, $u: \mathbb{R}_{+} \rightarrow \mathbb{R}_{+}$is continuously differentiable, strictly increasing, and strictly concave. Contingent on the observed dividend payment $\mathbf{y}_{t} \in \mathbf{Y}$, the Choquet expected utility of period $t$ agent's asset holding choice is given $\operatorname{as}^{9}$

$$
\begin{aligned}
& E\left[U\left(\mathbf{z}_{t+1}\right), \nu_{t+1, \ldots}\left(d\left(\mathbf{y}_{t+1}, \ldots\right) \mid \mathbf{y}_{t}\right)\right] \\
= & u\left(c\left(\mathbf{y}_{t}, \mathbf{p}_{t}, \mathbf{z}_{t}, \mathbf{z}_{t+1}\right)\right)+E\left[\sum_{s=t+1}^{\infty} \beta^{s-t} u\left(c\left(\mathbf{y}_{s}, \mathbf{p}_{s}, \mathbf{z}_{s}, \mathbf{z}_{s+1}\right)\right), \nu_{t+1, \ldots}\left(d\left(\mathbf{y}_{t+1}, \ldots\right) \mid \mathbf{y}_{t}\right)\right] .
\end{aligned}
$$

Let us, somewhat informally, describe this economy as a repeated Bayesian game such that the period $t=1,2, \ldots$ agents are different players whose beliefs about "nature's moves" are given as conditional neo-additive beliefs. A strategy of a period $t$ agent is then any $\mathcal{F}_{t}$-measurable function $\mathbf{f}_{t+1}: \mathbf{Y} \rightarrow[0,1]^{k}$; that is, a player's strategy maps the set of possible observation into the set of possible asset holdings at period $t+1$. Since consumption in time periods $s>t+1$ is irrelevant to the first-order conditions of the

\footnotetext{
${ }^{9}$ Notice that this definition of the Choquet expected utility of an uncertain infinite consumption stream coincides for the multiple-prior framework with definition (2.4.3) in Epstein and Wang (1994).
} 
maximization problem (35), we obtain the following characterization of an equilibrium under the assumptions that, firstly, all period $t$ agents choose mutually best responses with respect to belief $\nu(\cdot \mid \cdot)$ and, secondly, at equilibrium prices demand equals supply in each time period.

Definition: The strategy profile $\left\{\mathbf{f}_{t+1}^{*}\right\}_{t=1,2 \ldots}$ and the price-sequence $\left\{\mathbf{p}_{t}^{*}\right\}_{t=1,2, \ldots}$ constitute an equilibrium of this economy if and only if, for all observations $\mathbf{y}_{t} \in \mathbf{Y}$,

$$
\begin{aligned}
\mathbf{f}_{t+1}^{*}\left(\mathbf{y}_{t}\right)= & \arg \max _{\mathbf{z}_{t+1} \in[0,1]^{k}} u\left(c\left(\mathbf{y}_{t}, \mathbf{p}_{t}^{*}, \mathbf{f}_{t}^{*}, \mathbf{z}_{t+1}\right)\right) \\
& +\beta E\left[u\left(c\left(\mathbf{y}_{t+1}, \mathbf{p}_{t+1}^{*}, \mathbf{z}_{t+1}, \mathbf{f}_{t+2}^{*}\right)\right), \nu\left(d \mathbf{y}_{t+1} \mid \mathbf{y}_{t}\right)\right]
\end{aligned}
$$

such that $\mathbf{p}_{t}^{*}$ and $\mathbf{p}_{t+1}^{*}$ ensure

$$
\mathbf{z}_{t+1}^{*}=\mathbf{z}_{t}^{*}
$$

for all $t$.

Because of the market-clearing condition (37), we know that in any equilibrium every period $t$ agent must optimally hold the initial endowment of assets; that is, $\mathbf{f}_{t}^{*}=\mathbf{1}$ for all $t$. To study the (subgame-perfect) equilibria of this economy, it therefore remains to characterize the prices which support this allocation. The assumptions A1 and A2 on beliefs combined with the bounded memory assumption allow us to consider a stationary situation in which every period $t$ agent, $t=0,1, \ldots$, faces the same maximization problem. As a consequence, we can restrict attention to stationary price functions as candidates for equilibrium price functions.

Observation 3. A stationary price function $\mathbf{p}^{*}: \mathbf{Y} \rightarrow \times_{j=1}^{k} \mathbb{R}_{+}$is an equilibrium price-function if and only if, for all $\mathbf{y}_{t}, \mathbf{y}_{t+1} \in \mathbf{Y}$,

$$
\begin{aligned}
\mathbf{f}_{t+1}^{*}= & \mathbf{1}=\arg \max _{\mathbf{z}_{t+1} \in[0,1]^{k}} u\left(c_{t}\left(\mathbf{y}_{t}, \mathbf{p}^{*}\left(\mathbf{y}_{t}\right), \mathbf{f}_{t}^{*}=\mathbf{1}, \mathbf{z}_{t+1}\right)\right) \\
& +\beta E\left[u\left(c_{t+1}\left(\mathbf{y}_{t+1}, \mathbf{p}^{*}\left(\mathbf{y}_{t+1}\right), \mathbf{z}_{t+1}, \mathbf{f}_{t+2}^{*}=\mathbf{1}\right)\right), \nu\left(d \mathbf{y}_{t+1} \mid \mathbf{y}_{t}\right)\right]
\end{aligned}
$$

for all $t$, whereby

$$
\begin{aligned}
& E\left[u\left(c_{t+1}\left(\mathbf{y}_{t+1}, \mathbf{p}^{*}\left(\mathbf{y}_{t+1}\right), \mathbf{z}_{t+1}, \mathbf{f}_{t+2}^{*}=\mathbf{1}\right)\right), \nu\left(d \mathbf{y}_{t+1} \mid \mathbf{y}_{t}\right)\right] \\
= & \delta_{\mathbf{y}_{t}}\left(\lambda \cdot \max u\left(c_{t+1}\left(\mathbf{y}_{t+1}, \mathbf{p}^{*}\left(\mathbf{y}_{t+1}\right), \mathbf{z}_{t+1}, \mathbf{f}_{t+2}^{*}=\mathbf{1}\right)\right)\right. \\
& +(1-\lambda) \cdot \min u\left(c_{t+1}\left(\mathbf{y}_{t+1}, \mathbf{p}^{*}\left(\mathbf{y}_{t+1}\right), \mathbf{z}_{t+1}, \mathbf{f}_{t+2}^{*}=\mathbf{1}\right)\right) \\
& +\left(1-\delta_{\mathbf{y}_{t}}\right) E\left[u\left(c_{t+1}\left(\mathbf{y}_{t+1}, \mathbf{p}^{*}\left(\mathbf{y}_{t+1}\right), \mathbf{z}_{t+1}, \mathbf{f}_{t+2}^{*}=\mathbf{1}\right)\right), \pi\left(d \mathbf{y}_{t+1} \mid \mathbf{y}_{t}\right)\right] .
\end{aligned}
$$

The following proposition constitutes the paper's main formal result. 
Proposition 1. There exists a unique continuous equilibrium price-function $\mathbf{p}^{*}: \mathbf{Y} \rightarrow$ $\times_{j=1}^{k} \mathbb{R}_{+}$such that, for $j=1, \ldots, k$,

$$
p_{j}^{*}(\mathbf{y})=\frac{f_{j}^{*}(\mathbf{y})}{u^{\prime}(\mathbf{y} \cdot \mathbf{1})}, \mathbf{y} \in \mathbf{Y}
$$

whereby $f_{j}^{*} \in C[\mathbf{Y}]$ is the unique fixed point of the operator $T: C[\mathbf{Y}] \rightarrow C[\mathbf{Y}]$ defined by

$$
\begin{aligned}
\left(T f_{j}\right)(\mathbf{y})= & \beta\left[\delta _ { \mathbf { y } } \left(\lambda \cdot u^{\prime}(\overline{\mathbf{y}} \cdot \mathbf{1}) \cdot \bar{y}_{j}+f_{j}(\overline{\mathbf{y}})\right.\right. \\
& \left.+(1-\lambda) \cdot u^{\prime}(\underline{\mathbf{y}} \cdot \mathbf{1}) \cdot \underline{y}_{j}+f_{j}(\underline{\mathbf{y}})\right) \\
& \left.+\left(1-\delta_{\mathbf{y}}\right) E\left[u^{\prime}\left(\mathbf{y}^{\prime} \cdot \mathbf{1}\right) \cdot y_{j}^{\prime}+f_{j}\left(\mathbf{y}^{\prime}\right), \pi\left(d \mathbf{y}^{\prime} \mid \mathbf{y}\right)\right]\right]
\end{aligned}
$$

for all $\mathbf{y} \in \mathbf{Y}$. Moreover, as an approximation for the fixed-point $f_{j}^{*}$ we have for any $f_{j} \in C[\mathbf{Y}]$

$$
\left\|T^{n} f_{j}-f_{j}^{*}\right\|_{\infty} \leq \beta^{n}\left\|f_{j}-f_{j}^{*}\right\|_{\infty} .
$$

\section{Risk-free rate and equity premium}

An intermediate step in the proof of Proposition 1 derives the following first-order conditions for the period $t$ agent's portfolio optimization problem

$$
\begin{aligned}
p_{j, t}^{*}\left(\mathbf{y}_{t}\right) \cdot u^{\prime}\left(\mathbf{y}_{t} \cdot \mathbf{1}\right)= & \beta\left[\delta _ { \mathbf { y } _ { t } } \left(\lambda \cdot u^{\prime}\left(\overline{\mathbf{y}}_{t+1} \cdot \mathbf{1}\right) \cdot\left(\bar{y}_{j, t+1}+p_{j}^{*}\left(\overline{\mathbf{y}}_{t+1}\right)\right)\right.\right. \\
& \left.+(1-\lambda) \cdot u^{\prime}\left(\underline{\mathbf{y}}_{t+1} \cdot \mathbf{1}\right) \cdot\left(\underline{y}_{j, t+1}+p_{j}^{*}\left(\underline{\mathbf{y}}_{t+1}\right)\right)\right) \\
& \left.+\left(1-\delta_{\mathbf{y}_{t}}\right) E\left[u^{\prime}\left(\mathbf{y}_{t+1} \cdot \mathbf{1}\right) \cdot\left(y_{j, t+1}+p_{j}^{*}\left(\mathbf{y}_{t+1}\right)\right), \pi\left(d \mathbf{y}_{t+1} \mid \mathbf{y}\right)\right]\right]
\end{aligned}
$$

for $j=1, \ldots, k$. Having established the existence of a unique equilibrium in Proposition 1, we can use the FOCs (43) to describe properties of this equilibrium. Of particular interest are, firstly, the equilibrium gross-return of a risk-free asset and, secondly, the difference between this risk-free rate and the expected equilibrium gross-return of a risky asset. To this end define the $\mathcal{F}_{t+1}$-measurable (adopted) random variables

$$
R_{j}=\frac{p_{j}^{*}\left(\mathbf{y}_{t+1}\right)+y_{j, t+1}}{p_{j}^{*}\left(\mathbf{y}_{t}\right)}
$$

and

$$
M=\beta \cdot \frac{u^{\prime}\left(\mathbf{y}_{t+1} \cdot \mathbf{1}\right)}{u^{\prime}\left(\mathbf{y}_{t} \cdot \mathbf{1}\right)}
$$

$R_{j}$ stands for the gross-return of asset $j$ at equilibrium prices $p_{j}^{*}$ and $M$ stands for the stochastic discount factor, (due to the stationarity of the problem we can drop the 
time indices). Furthermore, let us introduce the following notational conventions for the values of the random gross-return and the random stochastic discount factor evaluated at the best, $\overline{\mathbf{y}}$, respectively worst, $\underline{\mathbf{y}}$, economic scenario

$$
\begin{aligned}
\bar{R}_{j} & =\frac{p_{j}^{*}(\overline{\mathbf{y}})+\bar{y}_{j}}{p_{j}^{*}\left(\mathbf{y}_{t}\right)} \text { and } \underline{R}_{j}=\frac{p_{j}^{*}(\underline{\mathbf{y}})+\underline{y}_{j}}{p_{j}^{*}\left(\mathbf{y}_{t}\right)}, \\
\bar{M} & =\beta \cdot \frac{u^{\prime}(\overline{\mathbf{y}} \cdot \mathbf{1})}{u^{\prime}\left(\mathbf{y}_{t} \cdot \mathbf{1}\right)} \text { and } \underline{M}=\beta \cdot \frac{u^{\prime}(\underline{\mathbf{y}} \cdot \mathbf{1})}{u^{\prime}\left(\mathbf{y}_{t} \cdot \mathbf{1}\right)} .
\end{aligned}
$$

Dividing equation (43) by its lhs terms and substituting $R_{j}, M, \bar{R}_{j}, \bar{M}, \underline{R}_{j}$, and $\underline{M}$ gives the following result.

Corollary 1. Expressed in terms of the gross-return of asset $j$ and the stochastic discount factor, the economy's equilibrium is characterized by the following conditions

$$
\begin{aligned}
1= & \delta_{\mathbf{y}_{t}}\left(\lambda \cdot \bar{M} \cdot \bar{R}_{j}+(1-\lambda) \cdot \underline{M} \cdot \underline{R}_{j}\right) \\
& +\left(1-\delta_{\mathbf{y}_{t}}\right) E\left[M \cdot R_{j}, \pi\left(d \mathbf{y}_{t+1} \mid \mathbf{y}_{t}\right)\right]
\end{aligned}
$$

for $j=1, \ldots, k$.

Note that whenever the neo-additive capacity reduces to an additive belief, i.e., $\delta_{\mathbf{y}_{t}}=0$, we are back to the familiar equilibrium asset-pricing relationship

$$
1=E\left[M \cdot R_{j}, \pi\left(d \mathbf{y}_{t+1} \mid \mathbf{y}_{t}\right)\right]
$$

of the standard EU economy. For the non-degenerate case $\delta_{\mathbf{y}_{t}}>0$, however, the period $t$ agent of the CEU Lucas economy has - in addition to this standard condition - also the best economic scenario, i.e., $\mathbf{Y}_{t+1}(\omega)=\overline{\mathbf{y}}$, and the worst economic scenario, i.e., $\mathbf{Y}_{t+1}(\omega)=\mathbf{y}$, on his mind. The difference between the CEU equilibrium condition (48) and the standard EU equilibrium condition (49) is the greater the greater the degree of ambiguity

$$
\delta_{\mathbf{y}_{t}}=\frac{\delta}{\delta+(1-\delta) \cdot \pi\left(\mathbf{y}_{t}\right)}
$$

is, which in turn decreases in the additive probability attached to the observation $\mathbf{y}_{t}$. That is, whenever the representative CEU agent observes dividend payments that are unlikely with respect to the additive part of his neo-additive prior, his equilibrium behavior will be rather different from the equilibrium behavior of the corresponding EU agent. In the plausible case that the additive part of the neo-additive prior attaches small probabilities to rather extreme economic outcomes, the CEU Lucas economy would thus generate - compared to the standard EU model - quite different implications on the 
risk-free rate and the equity premium whenever agents observe rather extreme economic outcomes.

In what follows we are going to investigate the impact of this preoccupation with extreme economic scenarios on the risk-free rate and the equity premium in the CEU Lucas economy.

\subsection{The risk-free rate}

For a risk-free asset, i.e., an asset that gives a constant gross return, we have that

$$
R_{f}=\bar{R}_{f}=\underline{R}_{f}
$$

so that the equilibrium condition (48) implies the following risk-free rate of return for the CEU Lucas economy

$$
R_{f}=\frac{1}{\delta_{\mathbf{y}_{t}}(\lambda \cdot \bar{M}+(1-\lambda) \cdot \underline{M})+\left(1-\delta_{\mathbf{y}_{t}}\right) E\left[M, \pi\left(d \mathbf{y}_{t+1} \mid \mathbf{y}_{t}\right)\right]}
$$

Observe now that the stochastic discount factor (45) is strictly decreasing in next period's dividend payments since $u^{\prime}$ is, by the concavity assumption, strictly decreasing so that

$$
\bar{M}<E\left[M, \pi\left(d \mathbf{y}_{t+1} \mid \mathbf{y}_{t}\right)\right]<\underline{M} .
$$

Thus, whenever there is some ambiguity, i.e., $\delta_{\mathbf{y}_{t}}>0$, the equilibrium risk-free rate of the CEU Lucas economy is smaller than in the standard EU economy if and only if

$$
\begin{aligned}
\lambda \cdot \bar{M}+(1-\lambda) \cdot \underline{M} & >E\left[M, \pi\left(d \mathbf{y}_{t+1} \mid \mathbf{y}_{t}\right)\right] \Leftrightarrow \\
\lambda & <\frac{\underline{M}-E\left[M, \pi\left(d \mathbf{y}_{t+1} \mid \mathbf{y}_{t}\right)\right]}{\underline{M}-\bar{M}} \in(0,1) .
\end{aligned}
$$

That is, the risk-free rate in the CEU Lucas economy is smaller (resp. greater) than the corresponding risk-free rate in a standard EU economy whenever the degree of optimism $\lambda$ is sufficiently small (resp. large).

\section{Corollary 2: "Risk-free rate"}

- An optimistic representative agent, who is preoccupied with the best possible economic scenario, gives rise to a rather large risk-free rate in the equilibrium of the CEU Lucas economy.

- In contrast, a pessimistic agent, who is preoccupied with the worst possible economic scenario, gives rise to a rather low risk-free rate in the equilibrium of the CEU Lucas economy. 
The findings of Corollary 2 are highly intuitive. Namely, an optimist who puts additional decision weight on the best case scenario - which is in the CEU Lucas economy the highest possible dividend payments of all assets - will require a rather high return from the risk-free asset in order to hold it together with the risky assets in his portfolio. Conversely, a pessimist who is preoccupied with the worst case scenario - in which the risky assets pay out the lowest possible dividends - wants to hold the risk-free asset even if it gives a rather low return since risky assets are not very attractive to him.

According to the risk-free rate puzzle (Mehra and Prescott 1985, 2003; Barberis and Thaler 2003), a realistically calibrated model of a Lucas-type economy yields a return on risk-free assets of about 5\% - 6\% compared to a real-world actual risk-free rate in the range of $1-2 \%$. It is an interesting question for future research of how far realistic values of the CEU Lucas economy parameters $\delta_{\mathbf{y}_{t}}, \lambda, \underline{M}$, and $\bar{M}$ may drive down the model-predicted risk-free rate from $5 \%-6 \%$ towards the empirically observed $1-2 \%$. If the answer is "not that much", this finding would support Simon Grant's and John Quiggin's claim that the asset return puzzles are caused by market imperfections rather than by preferences since even the introduction of ambiguity attitudes could then not nearly account for the risk-free rate puzzle. If, however, the answer is "quite a bit", this finding would support the notion that there is a preference-based explanation of the asset return puzzles in terms of ambiguity attitudes that are resolved in a pessimistic way.

\subsection{The equity premium}

For the expected rate of return of a risky asset $j$, the equilibrium condition (48) implies

$$
\begin{aligned}
& E\left[R_{j}, \pi\left(d \mathbf{y}_{t+1} \mid \mathbf{y}_{t}\right)\right] \\
= & -\frac{\operatorname{Cov}\left[M, R_{j}, \pi\left(d \mathbf{y}_{t+1} \mid \mathbf{y}_{t}\right)\right]}{E\left[M, \pi\left(d \mathbf{y}_{t+1} \mid \mathbf{y}_{t}\right)\right]}+\frac{1-\delta_{\mathbf{y}_{t}}\left(\lambda \cdot \bar{M} \cdot \bar{R}_{j}+(1-\lambda) \cdot \underline{M} \cdot \underline{R}_{j}\right)}{\left(1-\delta_{\mathbf{y}_{t}}\right) E\left[M, \pi\left(d \mathbf{y}_{t+1} \mid \mathbf{y}_{t}\right)\right]} .
\end{aligned}
$$

The equity-premium for the CEU Lucas economy, i.e., the difference between the expected return of a risky asset (56) and the risk-free rate (52), is thus given as

$$
\begin{aligned}
& E\left[R_{j}, \pi\left(d \mathbf{y}_{t+1} \mid \mathbf{y}_{t}\right)\right]-R_{f} \\
= & -\frac{\operatorname{Cov}\left[M, R_{j}, \pi\left(d \mathbf{y}_{t+1} \mid \mathbf{y}_{t}\right)\right]}{E\left[M, \pi\left(d \mathbf{y}_{t+1} \mid \mathbf{y}_{t}\right)\right]} \\
& +\frac{1}{\left(1-\delta_{\mathbf{y}_{t}}\right) E\left[M, \pi\left(d \mathbf{y}_{t+1} \mid \mathbf{y}_{t}\right)\right]} \\
& -\frac{1}{\delta_{\mathbf{y}_{t}}(\lambda \cdot \bar{M}+(1-\lambda) \cdot \underline{M})+\left(1-\delta_{\mathbf{y}_{t}}\right) E\left[M, \pi\left(d \mathbf{y}_{t+1} \mid \mathbf{y}_{t}\right)\right]} \\
& -\frac{\delta_{\mathbf{y}_{t}}\left(\lambda \cdot \bar{M} \cdot \bar{R}_{j}+(1-\lambda) \cdot \underline{M} \cdot \underline{R}_{j}\right)}{\left(1-\delta_{\mathbf{y}_{t}}\right) E\left[M, \pi\left(d \mathbf{y}_{t+1} \mid \mathbf{y}_{t}\right)\right]} .
\end{aligned}
$$


At first, verify that the familiar formula for the equity premium of the standard EU economy

$$
E\left[R_{j}, \pi\left(d \mathbf{y}_{t+1} \mid \mathbf{y}_{t}\right)\right]-R_{f}=-\frac{\operatorname{Cov}\left[M, R_{j}, \pi\left(d \mathbf{y}_{t+1} \mid \mathbf{y}_{t}\right)\right]}{E\left[M, \pi\left(d \mathbf{y}_{t+1} \mid \mathbf{y}_{t}\right)\right]}
$$

obtains as the special case of the CEU Lucas economy iff $\delta_{\mathbf{y}_{t}}=0$, i.e., iff there is no ambiguity. To interpret the equity-premium for the non-degenerate case where the agents express ambiguity, i.e., $\delta_{\mathbf{y}_{t}}>0$ observe that the term

$$
\frac{1}{\left(1-\delta_{\mathbf{y}_{t}}\right) E\left[M, \pi\left(d \mathbf{y}_{t+1} \mid \mathbf{y}_{t}\right)\right]}-\frac{1}{\delta_{\mathbf{y}_{t}}(\lambda \cdot \bar{M}+(1-\lambda) \cdot \underline{M})+\left(1-\delta_{\mathbf{y}_{t}}\right) E\left[M, \pi\left(d \mathbf{y}_{t+1} \mid \mathbf{y}_{t}\right)\right]}
$$

in (57) is always strictly positive since

$$
\delta_{\mathbf{y}_{t}}(\lambda \cdot \bar{M}+(1-\lambda) \cdot \underline{M})>0 .
$$

As a consequence, the equity premium of the CEU Lucas economy (57) is greater than the equity premium of the standard EU economy (58) whenever the term

$$
\frac{\delta_{\mathbf{y}_{t}}\left(\lambda \cdot \bar{M} \cdot \bar{R}_{j}+(1-\lambda) \cdot \underline{M} \cdot \underline{R}_{j}\right)}{(1-\delta) E\left[M, \pi\left(d \mathbf{y}_{t+1} \mid \mathbf{y}_{t}\right)\right]}
$$

in (57) is sufficiently small (i.e., smaller than (59)). This is the case for a pessimistic decision-maker (low $\lambda$ ) and a sufficiently low worst-case return-rate $\underline{R}_{j}$ of asset $j$. Conversely, the equity premium of the CEU Lucas economy (57) is smaller than (58) whenever (61) is sufficiently large. This is the case if the decision-maker is rather optimistic (high $\lambda$ ) and the best-case return-rate $\bar{R}_{j}$ of asset $j$ is rather high.

The following corollary collects these intuitive relationships.

\section{Corollary 3: "Equity premium"}

- An optimistic representative agent, who is preoccupied with the best possible economic scenario, gives rise to a rather low equity premium for a risky asset in the equilibrium of the CEU Lucas economy if this asset has a high best-case return.

- In contrast, a pessimistic representative agent, who is preoccupied with the worst possible economic scenario, gives rise to a rather large equity premium for a risky asset in the equilibrium of the CEU Lucas economy if this asset has a low worst-case return.

To sum up: The assumption of a CEU Lucas economy with a representative agent who resolves his ambiguity in a rather pessimistic way gives rise to a lower risk-free 
rate and larger equity premium than in the standard EU economy. Moreover, the differences to the standard EU risk-free rate and equity premium are the greater the more unlikely - from an ex ante perspective - the observation is that the agent makes about the economy's performance.

\section{Concluding remarks and outlook}

This paper studies a Lucas fruit-tree economy under the assumption that the agents are Choquet decision makers who have the best and the worst economic scenario on their minds. As the main insights obtained, the paper, firstly, establishes conditions which ensure the existence of a unique equilibrium price function and, secondly, it characterizes the resulting risk-free rate and the equity premium for this CEU Lucas economy. The applied equilibrium concept takes account of the fact that the agents in the CEU Lucas economy are dynamically inconsistent. In particular, the considered equilibrium concept combines the market clearing condition of general equilibrium theory with the concept of a subgame-perfect Bayesian Nash equilibrium with respect to conditional neo-additive beliefs. The conditional neo-additive beliefs of all time period agents are thereby generated by a time-homogenous stochastic process with restricted memory.

Based on the original Lucas fruit-tree economy formulated for EU agents with rational expectations in the sense of Muth (1961), Mehra and Prescott (1985) study a model with a normally distributed consumption growth rate process and CRRA utility for which they observe the so-called risk-free rate and equity premium puzzles. According to these puzzles, a realistically calibrated model implies, firstly, a much higher return of a riskfree asset and, secondly, a much lower difference in (expected) gross returns between a risky and a risk-free asset than observed in the empirical data. Although there is no productivity growth in the CEU Lucas economy studied in this paper, the discussion of the risk-free rate and the equity premium arising in the CEU Lucas economy indicates that the introduction of $\mathrm{CEU}$ preferences to consumption based asset pricing models may open an interesting avenue for future research with respect to asset return puzzles.

Although any thorough empirical discussion of these asset pricing puzzles is beyond the scope of the present paper, it would be a desirable avenue for future research to generalize this paper's model to an economy with normally distributed consumption growth that can be calibrated with realistic parameter-values and tested against empirical data. Since the assumption of a sufficiently pessimistic representative agent generates a lower risk-free rate and a higher equity premium for the CEU Lucas economy than for the corresponding standard EU economy, there is the prospect that the combination of am- 
biguity with pessimism may contribute towards a preference-based explanation of asset return puzzles which was not available for mere risk-aversion under the EU approach.

Given that unrealistically high levels of risk-aversion could resolve asset return puzzles within the standard EU framework, it may not be too surprising that sufficiently high degrees of pessimism might do the same job within a decision theoretic framework under ambiguity. Furthermore, pessimistic neo-additive capacities can be reinterpreted for non-extreme events (i.e., non-null events whose complements are also non-null) as subjective additive probabilities that overweight, compared to the objective counterpart, the worst-case scenario. One crucial difference between the CEU agents with ambiguous beliefs, on the one hand, and EU agents with subjective beliefs, on the other hand, however, is that pessimism under ambiguity offers a decision theoretic explanation why agents might overweight bad outcomes. Another crucial difference concerns the fact that Bayesian learning within a neo-additive framework does no longer result in convergence of neo-additive beliefs to their objective counterparts (Zimper 2009, 2011; Zimper and Ludwig 2009). As a consequence, the standard justification of Muth's (1961) rational expectations hypothesis - according to which subjective beliefs coincide with their objective counterparts for Bayesian learners in the long run - does not apply to the CEU Lucas economy. That is, even if we endow a period $t$ agent of the CEU Lucas economy with an arbitrarily large memory about past statistical information, his neo-additive beliefs would still be bounded away from the objective probabilities that generated this statistical information when he updates his beliefs through Bayesian learning. In this sense, the CEU Lucas economy considered in this paper adds something conceptual new to the literature on consumption-based asset pricing models that cannot be captured by standard EU models. 


\section{Appendix I: Decision theoretic preliminaries}

\section{Sure thing principle, consequentialism, and dynamic consistency}

Throughout the text let us restrict attention to a decision-theoretic framework that reduces in the standard way dynamic decision situations to a static Savage framework such that ex ante and ex post preferences are defined over Savage acts; that is, we assume that the reduction principle holds (Siniscalchi 2011). For such a framework Epstein and Schneider (2003) describe a decision theoretic model, i.e., the rectangular MMEU model, that combines some notion of ambiguity attitudes with dynamic consistency. Since the rectangular MMEU model also satisfies consequentialism, it follows from existing results in the decision theoretic literature (Ghirardato 2002, Epstein and Le Breton 1993) that this MMEU model must somehow violate universalism to the effect that both principles, consequentialism and dynamic consistency, cannot simultaneously hold at all events. In the remainder of this subsection I make this argument more precise by showing in which specific sense the rectangular MMEU model violates universalism.

To be more precise consider some state space $\Omega$ and some $\sigma$-algebra $\mathcal{F}$ which contains all events that are relevant to the decision maker in the sense that he attaches probabilities to these events, (for example, we might simply follow Savage (1954) and assume that $\mathcal{F}$ is given as the power-set $2^{\Omega}$ ). In a next step, let us follow Epstein and Schneider (2003) and fix some partition $\Pi$ of $\Omega$ whereby $\sigma(\Pi) \subseteq \mathcal{F}$ denotes the $\sigma$-algebra generated by $\Pi$. The events in $\sigma(\Pi)$ are interpreted as the events that are ex post observable by the decision maker. In what follows we will define behavioral principles restricted to the events in $\sigma(\Pi)$ whereby the principle of universalism is satisfied if and only if $\sigma(\Pi)=\mathcal{F}$. Intuitively speaking, universalism means that the behavioral principles will hold at all relevant events, i.e., at all events included in the largest possible information structure $\sigma(\Pi)=\mathcal{F}$, to the effect that the resulting decision theoretic model will be invariant with respect to any particularly fixed information structure $\sigma(\Pi)$.

Recall that a Savage act $f$ maps $\Omega$ into a set of consequences $X$, i.e., $f: \Omega \rightarrow X$. Further, consider any finite partition $\left\{B_{1}, \ldots, B_{n}\right\}$ of $\Omega$ such that $B_{i} \in \mathcal{F}$ for $i=1, . ., n$. Given Savage acts $f^{1}, \ldots, f^{n}$, denote by $f_{B_{1}}^{1} \ldots f_{B_{n}}^{n}: \Omega \rightarrow X$ the Savage act such that

$$
f_{B_{1}}^{1} \ldots f_{B_{n}}^{n}(\omega)=\left\{\begin{array}{cc}
f^{1}(\omega) & \text { for } \omega \in B_{1} \\
\ldots & \ldots \\
f^{n}(\omega) & \text { for } \omega \in B_{n} .
\end{array}\right.
$$

For complements $B, \neg B \in \mathcal{F}$ and acts $f, g$, let us simply write $f_{B} g$ instead of $f_{B} g_{\neg B}$. Ex ante preferences over Savage acts, denoted $\succeq$, are interpreted as the decision maker's preferences before he receives any information. In contrast, ex post preferences over 
Savage acts, denoted $\succeq_{B}$, are interpreted as preferences conditional on $B$, i.e., after the decision maker has observed the occurrence of some non-null event $B \in \mathcal{F}$. It is assumed that $\succeq$ as well as $\succeq_{B}$ impose a weak order on all Savage acts.

Definition: $\sigma(\Pi)$-consequentialism. For all Savage acts $f, g, h, h^{\prime}, h^{\prime \prime}, h^{\prime \prime \prime}$ and all non-null events $B \in \sigma(\Pi)$, the following condition holds for ex post preferences:

$$
f_{B} h \succeq_{B} g_{B} h^{\prime} \Leftrightarrow f_{B} h^{\prime \prime} \succeq_{B} g_{B} h^{\prime \prime \prime}
$$

The notion of consequentialism in the context of decision trees under risk goes back to Hammond (1989) and Machina (1989) whereby Burks (1977) already refers to the same concept as "invariance axiom" (see the formal definition at p. 268, Burks 1977). The above definition is closest to Machina's interpretation once the reduction principle is applied to decision trees:

"The philosophy behind this approach [=consequentialism; the author] is that the uncertainty that was involved in the rest of the tree, as represented by the probabilities at the snipped-off chance nodes and the planned choices at the snipped-off choice nodes, is now irrelevant and should be treated as if it never existed." (p. 1641)

In other words, (63) states that it should not matter for ex post preferences conditional on observation $B$ whatever consequences, governed either by $h, h^{\prime}, h^{\prime \prime}$, or $h^{\prime \prime \prime}$, might have happened outside of $B$. Innocuous and straightforward as this dynamic principle may appear, Machina (1989) argues strongly against its appeal for non-EU decision making. Nevertheless, consequentialism is, e.g., satisfied for the CEU decision makers of my model as well as for the MMEU decision makers in Epstein and Schmeidler (2003).

Definition: $\sigma(\Pi)$-dynamic consistency. For all Savage acts $f, g, h$ and all non-null events $B \in \sigma(\Pi)$, the following condition holds for the relationship between ex ante and ex post preferences:

$$
f_{B} h g_{B} h \Leftrightarrow f_{B} h \succeq_{B} g_{B} h
$$

Definition: $\sigma(\Pi)$-sure thing principle. For all Savage acts $f, g, h, h^{\prime}$ and all events $B \in \sigma(\Pi)$, the following condition holds for ex ante preferences: 


$$
f_{B} h \succeq g_{B} h \Rightarrow f_{B} h^{\prime} \succeq g_{B} h^{\prime} .
$$

The following observation basically restates an implication of Lemma 2 in Ghirardato (2002) for our slightly different definitions of consequentialism and dynamic consistency.

Observation 2. $\sigma(\Pi)$-consequentialism (63) combined with $\sigma(\Pi)$-dynamic consistency (64) implies the $\sigma(\Pi)$-sure thing principle (65) under reduction.

Proof: Consider any non-null $B \in \sigma(\Pi)$. Observe that

$$
\begin{aligned}
f_{B} h & \succeq g_{B} h \\
& \Rightarrow f_{B} h \succeq_{B} g_{B} h \text { by }(64) \\
& \Rightarrow f_{B} h^{\prime} \succeq_{B} g_{B} h^{\prime} \text { by }(63) \\
& \Rightarrow f_{B} h^{\prime} \succeq g_{B} h^{\prime} \text { by }(64),
\end{aligned}
$$

which proves the claim.

Next consider the following version of dynamic consistency restricted to events in $\sigma(\Pi)$ as introduced in Epstein and Schneider (2003) for their rectangular MMEU model.

Definition: Epstein and Schneider (E-S) $\sigma(\Pi)$-dynamic consistency. Given any finite partition $B_{1}, \ldots, B_{n}$ of $\Omega$ such that $B_{i} \in \sigma(\Pi)$ for $i=1, . ., n$. For all Savage acts $f^{1}, \ldots, f^{n}$ and $g^{1}, \ldots, g^{n}$, the following condition holds:

$$
f^{1} \succeq_{B_{1}} g^{1}, \ldots, f^{n} \succeq_{B_{n}} g^{n} \Rightarrow f_{B_{1}}^{1} \ldots f_{B_{n}}^{n} \succeq g_{B_{1}}^{1} \ldots g_{B_{n}}^{n},
$$

whereby $\succeq$ is strict iff at least one $\succeq_{B_{i}}$ is strict.

Observation 3. $\sigma(\Pi)$-consequentialism (63) combined with E-S $\sigma(\Pi)$-dynamic consistency (66) implies the $\sigma(\Pi)$-sure thing principle (65) under reduction.

Proof: By E-S dynamic $\sigma(\Pi)$-consistency, we have for any non-null $B \in \sigma(\Pi)$

$$
f_{B} h \succeq_{B} g_{B} h, h_{B^{c}} f \succeq_{B^{c}} h_{B^{c}} g \Rightarrow f_{B} h \succeq g_{B} h .
$$

Furthermore, observe that

$$
\begin{aligned}
h_{\neg B} f & \succeq{ }_{\neg B} h_{\neg B} f \text { by reflexivity of } \succeq_{\neg B} \\
& \Leftrightarrow h_{\neg B} f \succeq_{\neg B} h_{\neg B} g \text { by (63), }
\end{aligned}
$$

so that

$$
h_{\neg B} f \succeq_{\neg B} h_{\neg B} g
$$


is always satisfied under our assumptions of weak order and consequentialism. Consequently, (67) is equivalent to

$$
f_{B} h \succeq_{B} g_{B} h \Rightarrow f_{B} h \succeq g_{B} h,
$$

which implies, by completeness,

$$
f_{B} h \succeq_{B} g_{B} h \Leftarrow f_{B} h \succeq g_{B} h .
$$

That is, under the assumptions of Observation 3, E-S $\sigma(\Pi)$-dynamic consistency is equivalent to $\sigma(\Pi)$-dynamic consistency (64) so that Observation 2 implies the result.

By Observation 3, the rectangular MMEU model of Epstein and Schneider (2003) satisfying $\sigma(\Pi)$-consequentialism as well as the reduction principle - satisfies the sure thing principle at all events in $\sigma(\Pi)$. As a consequence, a violation of the sure thing principle is restricted to events that are not observable by the decision maker to the effect that ex ante ambiguity attitudes are governed by the decision maker's ex post information structure.

\section{Bayesian updating of non-additive probability measures}

An update rule specifies how the ex ante preference ordering $\succeq$ determines, for all nonnull $B \in \mathcal{F}$, the ex post preference ordering $\succeq_{B}$. In what follows I restrict attention to $B, h$-Bayesian update rules in the sense that there exists for every non-null $B \in \mathcal{F}$ and every pair of Savage acts $f, g$ some Savage act $h$ such that

$$
f_{B} h \succeq g_{B} h \text { implies } f_{B} h^{\prime} \succeq_{B} g_{B} h^{\prime \prime} \text { for all } h^{\prime}, h^{\prime \prime} .
$$

Such update rules obviously satisfy consequentialism since the ex post preferences are independent of consequences that happened outside of observation $B .{ }^{10}$

In case the sure thing principle is satisfied, the specification of $h$ in (68) would not matter for deriving ex post preferences. In the case of CEU preferences, however, different specifications of $h$ in (68) result in different ways of updating ex ante CEU into ex post CEU preferences. That is, for the CEU framework there exist several perceivable ways of defining a conditional capacity $\nu(\cdot \mid B)$ such that

$$
f \succeq_{B} g \Leftrightarrow E[U(f), \nu(\cdot \mid B)] \geq E[U(g), \nu(\cdot \mid B)]
$$

even if we restrict attention to consequentialist Bayesian update rules. For example, Gilboa and Schmeidler (1993) consider the interesting benchmark cases of the optimistic

\footnotetext{
${ }^{10}$ For an update rule for non-additive probability measures that violates consequentialism but satisfies dynamic consistency, see Sarin and Wakker (1998a).
} 
and pessimistic update rules where $h$ corresponds to the constant act giving the worst, respectively best, possible consequence. The corresponding definitions of conditional capacities are

$$
\nu(A \mid B)=\frac{\nu(A \cap B)}{\nu(B)} .
$$

for the optimistic and

$$
\nu(A \mid B)=\frac{\nu(A \cup \neg B)-\nu(\neg B)}{1-\nu(\neg B)} .
$$

for the pessimistic update rule.

In the present paper I restrict attention to the so-called full (or generalized) Bayesian update rule

$$
\nu(A \mid B)=\frac{\nu(A \cap B)}{\nu(A \cap B)+1-\nu(A \cup \neg B)},
$$

which results when $h$ in (68) is given as the so-called conditional certainty equivalent of $g$ on $B$, i.e., $h$ is the constant act such that $g \sim_{B} h$ (Eichberger, Grant, and Kelsey 2006). Compared to (70) and (71), the full Bayesian update rule is less extreme and arguably more realistic. Furthermore, applied to a neo-additive capacity (5), it has the advantage that the pessimism parameter remains constant throughout the updating process (see Observation 2). 


\section{Appendix II: Mathematical proofs}

Proof of Observation 1: By an argument in Schmeidler (1986), it suffices to restrict attention to a non-negative valued random variable $f$ so that

$$
E[f, \nu]=\int_{0}^{+\infty} \nu(\{\omega \in \Omega \mid f(\omega) \geq z\}) d z,
$$

which is equivalent to

$$
E[f, \nu]=\int_{\min f}^{\max f} \nu(\{\omega \in \Omega \mid f(\omega) \geq z\}) d z
$$

since the range of $f$ is closed and bounded. We consider a partition $P_{n}, n=1,2, \ldots$, of $\Omega$ with members

$$
A_{n}^{k}=\left\{\omega \in \Omega \mid a_{k, n}<f(\omega) \leq b_{k, n}\right\} \text { for } k=1, \ldots, 2^{n}
$$

such that

$$
\begin{aligned}
a_{k, n} & =[\max f-\min f] \cdot \frac{(k-1)}{2^{n}}+\min f \\
b_{k, n} & =[\max f-\min f] \cdot \frac{k}{2^{n}}+\min f .
\end{aligned}
$$

Define the step functions $a_{n}: \Omega \rightarrow \mathbb{R}$ and $b_{n}: \Omega \rightarrow \mathbb{R}$ such that, for $\omega \in A_{n}^{k}, k=1, \ldots, 2^{n}$,

$$
\begin{aligned}
& a_{n}(\omega)=a_{k, n} \\
& b_{n}(\omega)=b_{k, n} .
\end{aligned}
$$

Obviously,

$$
E\left[a_{n}, \nu\right] \leq E[f, \nu] \leq E\left[b_{n}, \nu\right]
$$

for all $n$ and

$$
\lim _{n \rightarrow \infty} E\left[b_{n}, \nu\right]-E\left[a_{n}, \nu\right]=0 .
$$

That is, $E\left[a_{n}, \nu\right]$ and $E\left[b_{n}, \nu\right]$ converge to $E[f, \nu]$ for $n \rightarrow \infty$. Furthermore, observe that

$$
\begin{aligned}
\min a_{n} & =\min f \text { for all } n, \text { and } \\
\max b_{n} & =\max f \text { for all } n .
\end{aligned}
$$

Since $\lim _{n \rightarrow \infty} \min b_{n}=\lim _{n \rightarrow \infty} \min a_{n}$ and $E\left[b_{n}, \pi\right]$ is continuous in $n$, we have

$$
\begin{aligned}
\lim _{n \rightarrow \infty} E\left[b_{n}, \nu\right] & =\delta\left(\lambda \lim _{n \rightarrow \infty} \max b_{n}+(1-\lambda) \lim _{n \rightarrow \infty} \min b_{n}\right)+(1-\delta) \lim _{n \rightarrow \infty} E\left[b_{n}, \pi\right] \\
& =\delta(\lambda \max f+(1-\lambda) \min f)+(1-\delta) E[f, \pi] .
\end{aligned}
$$


In order to prove Observation 1, it therefore remains to be shown that, for all $n$,

$$
E\left[b_{n}, \nu\right]=\delta\left(\lambda \max b_{n}+(1-\lambda) \min b_{n}\right)+(1-\delta) E\left[b_{n}, \pi\right]
$$

Since $b_{n}$ is a step function, the Choquet expectation of $b_{n}$ with respect to $\nu$ becomes

$$
\begin{aligned}
E\left[b_{n}, \nu\right] & =\sum_{A_{n}^{k} \in P_{n}} \nu\left(A_{n}^{2^{n}} \cup \ldots \cup A_{n}^{k}\right) \cdot\left(a_{k, n}-a_{k-1, n}\right) \\
& =\sum_{A_{n}^{k} \in P_{n}} a_{k, n} \cdot\left[\nu\left(A_{n}^{2^{n}} \cup \ldots \cup A_{n}^{k}\right)-\nu\left(A_{n}^{2^{n}} \cup \ldots \cup A_{n}^{k-1}\right)\right],
\end{aligned}
$$

implying for a neo-additive capacity

$$
\begin{aligned}
E\left[b_{n}, \nu\right]= & \max b_{n}\left[\delta \lambda+(1-\delta) \pi\left(A_{n}^{2^{n}}\right)\right]+\sum_{k=2}^{2^{n}-1} a_{k, n}(1-\delta) \pi\left(A_{n}^{k}\right) \\
& +\min b_{n}\left[1-\delta \lambda-(1-\delta) \sum_{k=2}^{2^{n}} \pi\left(A_{n}^{k}\right)\right] \\
= & \delta \lambda \max b_{n}+(1-\delta) \sum_{k=1}^{2^{n}} a_{k, n} \pi\left(A_{n}^{k}\right)+\min b_{n}[\delta-\delta \lambda] \\
= & \delta\left(\lambda \max b_{n}+(1-\lambda) \min b_{n}\right)+(1-\delta) E\left[b_{n}, \pi\right] .
\end{aligned}
$$

Proof of Proposition 1: Since period $t+1$ consumption is strictly increasing in $\mathbf{y}_{t+1}$ for any given $\mathbf{z}_{t+1}$, we have by the existence of $\max \mathbf{Y}_{t+1}$ and $\min \mathbf{Y}_{t+1}$ that

$$
\begin{aligned}
& \max u\left(c_{t+1}\left(\mathbf{y}_{t+1}, \mathbf{p}^{*}\left(\mathbf{y}_{t+1}\right), \mathbf{z}_{t+1}, \mathbf{f}_{t+2}^{*}=\mathbf{1}\right)\right)=u\left(c_{t+1}\left(\overline{\mathbf{y}}_{t+1}, \mathbf{p}^{*}\left(\overline{\mathbf{y}}_{t+1}\right), \mathbf{z}_{t+1}, \mathbf{f}_{t+2}^{*}=\mathbf{1}\right)\right) \\
& \min u\left(c_{t+1}\left(\mathbf{y}_{t+1}, \mathbf{p}^{*}\left(\mathbf{y}_{t+1}\right), \mathbf{z}_{t+1}, \mathbf{f}_{t+2}^{*}=\mathbf{1}\right)\right)=u\left(c_{t+1}\left(\underline{\mathbf{y}}_{t+1}, \mathbf{p}^{*}\left(\underline{\mathbf{y}}_{t+1}\right), \mathbf{z}_{t+1}, \mathbf{f}_{t+2}^{*}=\mathbf{1}\right)\right)
\end{aligned}
$$

whereby

$$
\overline{\mathbf{y}}_{t+1}=\overline{\mathbf{y}} \text { and } \underline{\mathbf{y}}_{t+1}=\underline{\mathbf{y}} \text { for all } t \text { and all } \mathbf{z}_{t+1} \text {. }
$$

Consequently, (39) becomes

$$
\begin{aligned}
& E\left[u\left(c_{t+1}\left(\mathbf{y}_{t+1}, \mathbf{p}^{*}\left(\mathbf{y}_{t+1}\right), \mathbf{z}_{t+1}, \mathbf{f}_{t+2}^{*}=\mathbf{1}\right)\right), \nu\left(d \mathbf{y}_{t+1} \mid \mathbf{y}_{t}\right)\right] \\
= & \delta_{\mathbf{y}_{t}}\left(\lambda \cdot u\left(c_{t+1}\left(\overline{\mathbf{y}}_{t+1}, \mathbf{p}^{*}\left(\overline{\mathbf{y}}_{t+1}\right), \mathbf{z}_{t+1}, \mathbf{f}_{t+2}^{*}=\mathbf{1}\right)\right)\right. \\
& +(1-\lambda) \cdot u\left(c_{t+1}\left(\underline{\mathbf{y}}_{t+1}, \mathbf{p}^{*}\left(\underline{\mathbf{y}}_{t+1}\right), \mathbf{z}_{t+1}, \mathbf{f}_{t+2}^{*}=\mathbf{1}\right)\right) \\
& +\left(1-\delta_{\mathbf{y}_{t}}\right) E\left[u\left(c_{t+1}\left(\mathbf{y}_{t+1}, \mathbf{p}^{*}\left(\mathbf{y}_{t+1}\right), \mathbf{z}_{t+1}, \mathbf{f}_{t+2}^{*}=\mathbf{1}\right)\right), \pi\left(d \mathbf{y}_{t+1} \mid \mathbf{y}_{t}\right)\right] .
\end{aligned}
$$


The corresponding FOCs of the maximization problem are therefore

$$
\begin{aligned}
p_{j, t}^{*}\left(\mathbf{y}_{t}\right) \cdot u^{\prime}\left(\mathbf{y}_{t} \cdot \mathbf{1}\right)= & \beta\left[\delta _ { \mathbf { y } _ { t } } \left(\lambda \cdot u^{\prime}\left(\overline{\mathbf{y}}_{t+1} \cdot \mathbf{1}\right) \cdot\left(\bar{y}_{j, t+1}+p_{j}^{*}\left(\overline{\mathbf{y}}_{t+1}\right)\right)\right.\right. \\
& \left.+(1-\lambda) \cdot u^{\prime}\left(\underline{\mathbf{y}}_{t+1} \cdot \mathbf{1}\right) \cdot\left(\underline{y}_{j, t+1}+p_{j}^{*}\left(\underline{\mathbf{y}}_{t+1}\right)\right)\right) \\
& \left.+\left(1-\delta_{\mathbf{y}_{t}}\right) E\left[u^{\prime}\left(\mathbf{y}_{t+1} \cdot \mathbf{1}\right) \cdot\left(y_{j, t+1}+p_{j}^{*}\left(\mathbf{y}_{t+1}\right)\right), \pi\left(d \mathbf{y}_{t+1} \mid \mathbf{y}\right)\right]\right]
\end{aligned}
$$

for $j=1, \ldots, k$. Since the problem is stationary, we have $\mathbf{p}_{t}^{*}(\mathbf{y})=\mathbf{p}^{*}(\mathbf{y})$ for all $\mathbf{y}$ and $t$ so that we may drop the time-indices. Consider now the operator $T$ defined in (41) and observe that $p_{j}^{*}: \mathbf{Y} \rightarrow \mathbb{R}$ exists and is unique if and only if $T$ has a unique fixed point $f_{j}^{*}=\left(T f_{j}^{*}\right)$ so that $p_{j}^{*}=\frac{f_{j}^{*}}{u^{\prime}}$. Since $\mathbf{Y}$ is compact, any real valued continuous function $f$ in $\mathbf{Y}$ is also bounded so that $f \in C[\mathbf{Y}]$ whereby $C[\mathbf{Y}]$ denotes the space of bounded real-valued continuous functions in $\mathbf{Y}$ endowed with the supremum norm $\|\cdot\|_{\infty}$. Recall that $C[\mathbf{Y}]$ is a complete metric space. By the Feller property, any function $T f_{j}: \mathbf{Y} \rightarrow \mathbb{R}$ is also continuous so that the operator $T$ maps the complete metric space $C[\mathbf{Y}]$ into itself. As a consequence, we can apply the contraction mapping theorem according to which there exists a unique fixed point of $T$ if there exists some number (=modulus) $c<1$ such that, for all functions $f, g \in C[\mathbf{Y}]$,

$$
\|T f-T g\|_{\infty} \leq c \cdot\|f-g\|_{\infty}
$$

By Theorem 5 in Blackwell (1965), if $T$ is monotone and satisfies, for all functions $f_{j}$ and any constant $a$,

$$
\left[T\left(f_{j}+a\right)\right](\mathbf{y}) \leq\left(T f_{j}\right)(\mathbf{y})+c \cdot a
$$

for some $c<1$, then $T$ is a contraction with modulus $c$. It is easy to see that (41) is monotone, i.e., $f_{j}(\mathbf{y}) \geq g_{j}(\mathbf{y})$ for all $\mathbf{y}$ implies $T f_{j} \geq T g_{j}$. In order to prove that $T$ is a contraction, it therefore remains to be shown that condition (79) is satisfied for some $c<1$. Observe that

$$
\begin{aligned}
\left(T f_{j}+a\right)(\mathbf{y})= & \beta\left[\delta _ { \mathbf { y } } \left(\lambda \cdot\left(u^{\prime}(\overline{\mathbf{y}} \cdot \mathbf{1}) \cdot \bar{y}_{j}+f_{j}(\overline{\mathbf{y}})+a\right)\right.\right. \\
& \left.+(1-\lambda) \cdot\left(u^{\prime}(\underline{\mathbf{y}} \cdot \mathbf{1}) \cdot \underline{y}_{j}+f_{j}(\underline{\mathbf{y}})+a\right)\right) \\
& \left.+\left(1-\delta_{\mathbf{y}}\right) E\left[u^{\prime}\left(\mathbf{y}^{\prime} \cdot \mathbf{1}\right) \cdot y_{j}^{\prime}+f_{j}\left(\mathbf{y}^{\prime}\right)+a, \pi\left(d \mathbf{y}^{\prime} \mid \mathbf{y}\right)\right]\right] \\
= & \left(T f_{j}\right)(\mathbf{y})+\beta\left[\delta_{\mathbf{y}}(\lambda \cdot a+(1-\lambda) \cdot a)+\left(1-\delta_{\mathbf{y}}\right) E\left[a, \pi\left(d \mathbf{y}^{\prime} \mid \mathbf{y}\right)\right]\right] \\
= & \left(T f_{j}\right)(\mathbf{y})+\beta \cdot a .
\end{aligned}
$$

Thus, set $c=\beta$ to see that (79) is satisfied whenever $\beta<1$. $\square$ 


\section{References}

Anscombe, F.J., and R.J. Aumann (1963) "A Definition of Subjective Probability" Annals of American Statistics 34, 199-205.

Bidarkota, P.V., and B.V. Dupoyet (2007) "The Impact of Fat Tails on Equilibrium Rates of Return and Term Premia" Journal of Economic Dynamics and Control 31, 887-905.

Bidarkota, P.V., Dupoyet, B.V., and J.H. McCulloch (2009) "Asset Pricing with Incomplete Information and Fat Tails" Journal of Economic Dynamics and Control 33, 1314-1331.

Bidarkota, P.V., and J.H. McCulloch (2003) "Consumption Asset Pricing with Stable Shocks: Exploring a Solution and its Implications for Mean Equity Returns" Journal of Economic Dynamics and Control 27, 399-421.

Blackwell, D. (1965) "Discounted Dynamic Programming" The Annals of Mathematical Statistics 36, 226-235.

Burks, A.W. (1977) Chance, Cause, Reason: An Inquiry into the Nature of Scientific Evidence The University of Chicago Press, Chicago

Campbell, J.Y. (2003) "Consumption Based Asset Pricing" in: G.M. Constantinides, M. Harris and R.M. Stulz, eds., Handbook of the Economics and Finance. Amsterdam: Elsevier, 808-887.

Chateauneuf, A., Eichberger, J., and S. Grant (2007) "Choice under Uncertainty with the Best and Worst in Mind: Neo-additive Capacities" Journal of Economic Theory $127,538-567$.

Cochrane, J.H. (2001) Asset Pricing Princeton University Press: Princeton.

Cohen, M., Gilboa, I., Jaffray J.Y., and D. Schmeidler (2000) "An Experimental Study of Updating Ambiguous Beliefs" Risk, Decision, and Policy 5, 123-133.

Duffie, D. (2001) Dynamic Asset Pricing Theory Princeton University Press: Princeton.

Eichberger, J., and D. Kelsey (1999) "E-Capacities and the Ellsberg Paradox" Theory and Decision 46, 107-140.

Eichberger, J., Grant, S., and D. Kelsey (2006) "Updating Choquet Beliefs" Journal of Mathematical Economics 43, 888-899. 
Ellsberg, D. (1961) "Risk, Ambiguity and the Savage Axioms" Quarterly Journal of Economics 75, 643-669.

Epstein, L.G. (1999) "A Definition of Uncertainty Aversion" The Review of Economic Studies 66, 579-608.

Epstein, L.G. and M. Schneider (2003) "Recursive Multiple-Priors" Journal of Economic Theory 113, 1-31.

Epstein, L.G. and T. Wang (1994) "Intertemporal Asset Pricing Under Knightian Uncertainty" Econometrica 62, 283-322.

Ghirardato, P. (2002) "Revisiting Savage in a Conditional World" Economic Theory 20, 83-92.

Ghirardato, P., and M. Marinacci (2002) "Ambiguity Made Precise: A Comparative Foundation" Journal of Economic Theory 102, 251-289.

Gilboa, I. (1987) "Expected Utility with Purely Subjective Non-Additive Probabilities" Journal of Mathematical Economics 16, 65-88.

Gilboa, I. and D. Schmeidler (1989) "Maxmin Expected Utility with Non-Unique Priors" Journal of Mathematical Economics 18, 141-153.

Gilboa, I., and D. Schmeidler (1993) "Updating Ambiguous Beliefs" Journal of Economic Theory 59, 33-49.

Grant, S., and J. Quiggin (2002) "The Risk Premium for Equity: Implications for Clinton's Proposed Diversification of the Social Security Fund" American Economic Review 92, 1104-1115.

Grant, S., and J. Quiggin (2003 "Public Investment and the Risk Premium for Equity" Economica 70, 1-18.

Grant, S., and J. Quiggin (2005) "What Does the Equity Premium Mean?" The Economist's Voice 2, Article 2.

Hammond, P.J. (1988) "Consequentialist Foundation for Expected Utility" Theory and Decision 25, 25-78.

Hansen, L.P., Sargent, T.J., and T.D. Tallarini (1999) "Robust Permanent Income and Pricing" The Review of Economic Studies 66, 873-907. 
Hansen, L.P., Sargent, T.J., Turmuhambetova, G. and N. Williams (2006) "Robust Control and Model Misspecification" Journal of Economic Theory 128, 45-90.

Kahneman, D., and A. Tversky (1979) "Prospect Theory: An Analysis of Decision under Risk" Econometrica 47, 263-291.

Kocherlakota, N. (1996) "The Equity Premium: It's Still a Puzzle " Jounral of Economic Literature $\mathbf{3 4}$, 42-71.

Liu, H. (2011) "Dynamic Portfolio Choice under Ambiguity and Regime Switching Mean Returns" Journal of Economic Dynamics and Control 35, 623-640.

Lucas, R.E.Jr. (1978) "Asset Prices in an Exchange Economy" Econometrica 46, 14291445.

Machina, M. (1989) "Dynamic Consistency and Non-Expected Utility Models of Choice Under Uncertainty" Journal of Economic Literature 27, 1622-1668.

Mehra, R., and E.C. Prescott (1985) "The Equity Premium: A Puzzle" Journal of Monetary Economics 15, 145-161.

Mehra, R., and E.C. Prescott (2003) "The Equity Premium in Retrospect" in: G.M. Constantinides, M. Harris and R.M. Stulz, eds., Handbook of the Economics and Finance. Amsterdam: Elsevier, 808-887.

Miao, J., and N. Wang (2011) "Risk, Uncertainty, and Option Exercise" Journal of Economic Dynamics and Control 35, 442-461.

Muth, J.F. (1961) "Rational Expectations and the Theory of Price Movements" Econometrica 29, 315-335.

Pires, C.P. (2002) "A Rule for Updating Ambiguous Beliefs" Theory and Decision 53, $137-152$.

Quiggin, J.P. (1981) "Risk Perception and Risk Aversion among Australian Farmers" Australian Journal of Agricultural Economics 25, 160-169.

Quiggin, J.P. (1982) "A Theory of Anticipated Utility" Journal of Economic Behavior and Organization 3, 323-343.

Sarin, R., and P.P. Wakker (1992) "A Simple Axiomatization of Nonadditive Expected Utility" Econometrica 60, 1255-1272. 
Sarin, R., and P.P. Wakker (1998a) "Revealed Likelihood and Knightian Uncertainty" Journal of Risk and Uncertainty 16, 223-250.

Sarin, R., and P.P. Wakker (1998b) "Dynamic Choice and NonExpected Utility" Journal of Risk and Uncertainty 17, 87-119.

Savage, L.J. (1954) The Foundations of Statistics, John Wiley and \& Sons, Inc.: New York, London, Sydney.

Schmeidler, D. (1986) "Integral Representation without Additivity" Proceedings of the American Mathematical Society 97, 255-261.

Schmeidler, D. (1989) "Subjective Probability and Expected Utility without Additivity" Econometrica 57, 571-587.

Siniscalchi, M. (2011) "Dynamic Choice under Ambiguity" Theoretical Economics 6, $379-421$.

Tokat, Y., Rachev, S.T., and E.S. Schwartz (2003) "The Stable Non-Gaussian Asset Allocation: A Comparison with the Classical Gaussian Approach" Journal of Economic Dynamics and Control 27, 937-969.

Tversky, A., and D. Kahneman (1992) "Advances in Prospect Theory: Cumulative Representations of Uncertainty" Journal of Risk and Uncertainty 5, 297-323.

Tversky, A., and P.P. Wakker (1995) "Risk Attitudes and Decision Weights" Econometrica 63, 1255-1280.

Wakker, P.P. (2001) "Testing and Characterizing Properties of Nonadditive Measures through Violations of the Sure-Thing Principle" Econometrica 69, 1039-1059.

Wakker, P.P (2004) "On the Composition of Risk Preference and Belief" Psychological Review 111, 236-241.

Wakker, P.P. (2010) Prospect Theory: For Risk and Ambiguity Cambridge University Press: Cambridge, UK.

Wakker, P.P, and A. Tversky (1993) "An Axiomatization of Cumulative Prospect Theory" Journal of Risk and Uncertainty 7, 147-176.

Zimper, A. (2009) "Half Empty, Half Full and Why We Can Agree to Disagree Forever" Journal of Economic Behavior and Organization 71, 283-299. 
Zimper, A. (2011) "Do Bayesians Learn their Way out of Ambiguity?" Decision Analysis forthcoming.

Zimper, A., and A. Ludwig (2009) "On Attitude Polarization under Bayesian Learning with Non-additive Beliefs" Journal of Risk and Uncertainty 39, 181-212. 